\title{
Stimuli to the Revision Process: The Case for Apex Vessels
}

\author{
Stimuli articles do not necessarily reflect the policies of the USPC or the USP Council of Experts
}

\author{
James Mann ${ }^{\mathrm{a}}$, Michael Cohen ${ }^{\mathrm{b} 1}$, Andreas Abend ${ }^{\mathrm{c}}$, Carrie Coutant ${ }^{\mathrm{d}}$, Lee Ashworth ${ }^{\mathrm{a} 1}$, Robert Shaw ${ }^{\mathrm{a} 1}$, \\ Gavin Reynolds ${ }^{\mathrm{a} 1}$, Ishai Nire, Vivek Shahf, Steven Shaw ${ }^{\mathrm{g}}$, Ashvin Patel ${ }^{\mathrm{g}}$, Xujin Lư ${ }^{\mathrm{h}}$, Vincent Cicale ${ }^{\mathrm{h}}$, \\ Meagan Mccallum ${ }^{\text {h }}$, Sanjaykumar Patelc, Josey Topolskic, Steve Prüferi, Irena Tomaszewskab2, \\ Alexandros Kourentas ${ }^{j}$, Martin Mueller-Zsigmondy $y^{j}$, Julian Williamsk ${ }^{k}$, Matthew Ainge ${ }^{k}$, Philippe \\ Berben', Anne Bouquelle', Bertil Abrahamsson ${ }^{\mathrm{a} 2}$, Anders Karlsson ${ }^{\mathrm{a} 2}$, Ria Varghese ${ }^{\mathrm{b2}}$, Fasheng Li $^{\mathrm{b} 3}$, \\ Amy Orce ${ }^{\mathrm{b} 4}$, Beverly Nickerson ${ }^{\mathrm{b} 4}$, Xi Shao $^{\mathrm{m}}$ \\ ${ }^{a 1}$ Oral Product Development, Pharmaceutical Technology \& Development, Operations, AstraZeneca, Macclesfield, UK. \\ ${ }^{b 1}$ Worldwide Research and Development, Global Chemistry and Manufacturing Controls, Pfizer Inc. Groton, CT. \\ 'Pharmaceutical Sciences, Merck \& Co., Inc., Rahway, NJ. \\ dLilly Research Laboratory, Lilly Corporate Center, Eli Lilly and Company, Indianapolis, IN. \\ ${ }^{e}$ Distek, Inc., North Brunswick, NJ. \\ fSotax Corp., Westborough, MA. \\ ${ }^{g}$ Teledyne Hanson Research, Chatsworth, CA. \\ ${ }^{h}$ Drug Product Development, Global Product Development \& Supply, Bristol-Myers Squibb, New Brunswick, NJ. \\ ${ }^{i}$ ERWEKA GmbH, Langen, Germany. \\ ${ }^{b 2}$ Worldwide Research and Development, Analytical Research and Development, Pfizer Inc., Sandwich, UK. \\ ${ }^{j} A R D$ Dissolution \& Biopharmaceutics, Novartis Pharma AG, Basel, Switzerland. \\ ${ }^{k}$ Agilent Technologies Inc., Craven Arms, Shropshire, UK. \\ 'Pharmaceutical Development, UCB Pharma SA, Braine l'Alleud, Belgium. \\ ${ }^{a 2}$ Oral Product Development, Pharmaceutical Technology \& Development, Operations, AstraZeneca, Gothenburg, Sweden. \\ ${ }^{b 3}$ Worldwide Research and Development, Analytical Research and Development, PharmSci \& PGS Statistics, Pfizer Inc. Groton, CT. \\ ${ }^{b 4}$ Worldwide Research and Development, Analytical Research and Development, Pfizer Inc. Groton, CT. \\ ${ }^{m}$ Analytical Research \& Development, Development Sciences, AbbVie, North Chicago, IL.
}

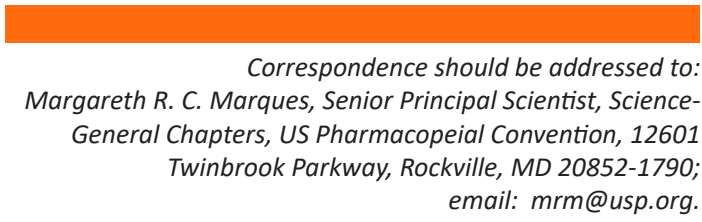

\section{ABSTRACT}

Apex vessels (previously known as PEAK vessels) are an important element of the dissolution scientist's toolbox and are frequently used in pharmaceutical drug product development settings. However, their use in development has not translated widely into use in the final approved quality control (QC) method. This Stimuli article aims to demonstrate the significant benefit of the apex vessel relative to the standard vessel in overcoming coning for formulations that contain dense insoluble excipients. Industrial case studies outline the benefits obtained by the apex vessel such as improved clinical relevance, more robust and discriminatory methods, and streamlined in vitro bridging strategies. Furthermore, to understand the impact of apex vessels produced by different dissolution bath manufacturers, an interlaboratory study was performed across 11 partners, which demonstrated minimal differences in dissolution performance between partners when a controlled protocol was executed. This was supplemented by a comparison between the different manufacturer designs using a computational fluid dynamic model, which showed no significant differences between manufacturers. This led to a manufacturer proposed specification for an apex vessel alongside a qualification procedure for the use of the vessels. It is the authors' intent by publishing this article that it will stimulate discussion leading to greater acceptance of the apex vessel such that it will be considered for a more prominent inclusion in future pharmacopeial chapters such as the US Pharmacopeia (USP) chapter The Dissolution Procedure: Development And Validation <1092> and ultimately inclusion into the USP chapter Dissolution $<711>$ and other harmonized pharmacopoeia as an alternative vessel to the standard 1-L vessel to be used when scientifically justified. 


\section{INTRODUCTION}

D issolution testing is an important tool used within the pharmaceutical industry to provide supportive evidence of the safety and efficacy of a drug product. The potential to link in vivo drug product performance to in vitro dissolution results in a high level of scrutiny by regulators of both the test method and acceptance criteria $(1,2)$. The most commonly used apparatus for oral solid dosage forms is the paddle apparatus, often known as the USP Apparatus 2; however, dissolution scientists understand that in the standard $1-\mathrm{L}$ vessel, there is a hydrodynamic liability that results in an artificially low rate and extent of release for formulations with certain characteristics. This phenomenon is known as coning, due to the presence of a cone of dense material piled at the bottom of the vessel in the region directly under the paddle. This region has been shown in computational fluid dynamic studies to be a region of heterogenous shear rate under typical operating conditions and dissolution rates can vary significantly when exposed to different shear environments $(3,4)$. Baxter et al. demonstrated that for three different tablet products, including the USP Prednisone Tablets RS, the dissolution rate was slower and with a lower extent of release for tablets positioned centrally compared with tablets positioned $2 \mathrm{~cm}$ off center.

Coning is reported to be a function of excipient and/or drug particle size, particle density, fluid viscosity, fluid density, apparatus configurations, and agitation strength (5). Under normal aqueous dissolution conditions (e.g., 500-1000 $\mathrm{mL}$ volume) using the typical pharmacopeial dissolution apparatus and paddle speed (e.g., $50 \mathrm{rpm}$ ), coning is generally related to the formulation composition.

Commonly used insoluble excipients such as certain grades of dicalcium phosphate or high amounts of microcrystalline cellulose, which have a high bulk density, are often implicated in the occurrence of coning. The implications of coning in dissolution methodology can often be far reaching. For example, if it is necessary during development to bridge from an early formulation such as a blend in capsule to a later tablet formulation that exhibits coning, then it may not be possible, using a pharmacopeial apparatus and a wholly in vitro strategy, to show similar dissolution performance. In such cases where dissolution profile similarity is required [e.g., Biopharmaceutics Classification System (BCS) biowaivers], coning is an artifact that may result in non-similarity, leading to the need for in vivo studies.

It has therefore been a subject of great interest to improve the hydrodynamic situation in the USP Apparatus 2 vessel for many years and minimize the impact of coning. PEAK vessels were introduced by VanKel (Cary, NC) (6) in the mid-1990s and were a promising option. Collins and Nair then demonstrated that the PEAK vessel increased the release profile and decreased variability due to the shape of the vessel minimizing any cone effect (7). This was then further investigated by Baxter et al., who demonstrated using computational fluid dynamic (CFD) modeling that the PEAK vessel was able to decrease the shear heterogeneity observed in a standard vessel in the locations where a tablet is most likely to reside during a dissolution test (8), i.e., directly under the paddle. Overall, the PEAK vessel has become an important part of the dissolution scientist's development toolbox but has seen very limited application in final commercial (i.e., approved) methods. A check of both the U.S. Food and Drug Administration (FDA) and USP dissolution database reveals only four mentions of PEAK vessels. First, in the FDA database, the atovaquone/proguanil hydrochloride product was added in 2006, which is an unusual method as it uses PEAK vessels and a dissolution medium containing $40 \%$ isopropanol. Both of these implementations were acknowledged by the regulator in the review paperwork as unconventional, but the use of PEAK vessels was explicitly called out to overcome the effect of coning. The remaining mentions come from the USP database where galantamine tablets, praziquantel tablets (for veterinary use), and aprepitant capsules are stated to have the dissolution performed with USP Apparatus 2 and PEAK vessels. For both the galantamine and aprepitant products, alternative methods are also provided that do not use PEAK vessels. This lack of uptake in use for approved dissolution methodology would suggest a barrier to acceptability. USP chapter $<711>$ on dissolution makes no reference to PEAK vessels; however, USP <1092>, the guidance chapter on dissolution method development, does refer to PEAK vessels and has had an expanded description added in the December 2020 update to now state "...may be useful to eliminate coning (mounding of material at the bottom of the vessel)". It was also in the December 2020 update that a change in the name from the trademarked PEAK to the generic name apex vessel was implemented, which was seen as a minor barrier to wider acceptability. The PEAK vessel name was trademarked and patented by VanKel (9), and the product line was subsequently acquired by Varian and later Agilent (Santa Clara, CA). Since the patent expired, the vessel design is available from other sources; however, the trademark is maintained by Agilent. Therefore, a more generic name was needed moving forward, and it is for this reason that the proposed name is apex vessels, which reflects the introduction of the inverted cone forming an

Dissolution' 
apex below the paddle. There is precedent for this; the Enhancer Cell was trademarked but introduced into the USP chapter Semisolid Drug Products-Performance Tests 〈1724〉 with a more generic term of "immersion cell". Moving forward in this Stimuli article, we will refer to apex vessels.

Within the International Consortium for Innovation and Quality in Pharmaceutical Development (IQ) dissolution working group, the apparent barriers to utilizing an apex vessel in both development and registration methods were discussed. In many cases, the noncompendial status of the apex vessel is perceived to be an additional regulatory risk in the overall development and registration of a drug product, such that use of apex vessels in a registration application is discouraged by internal stakeholders. Further barriers identified were a) that no specification exists to ensure equivalence between different manufacturers of apex vessels and b) no agreed procedure exists for qualification of a dissolution system that was switched from a standard to an apex vessel. Therefore, in order to facilitate any future inclusion in a pharmacopeia, the IQ dissolution working group, along with the instrumentation subgroup of the American Association of Pharmaceutical Scientists (AAPS) In Vitro Release and Dissolution Testing (IVRDT) community determined that the best path forward was to both demonstrate the benefit of apex vessels through the use of industrial case studies as well as to determine a specification and qualification procedure for an apex vessel. This will ensure that any method developed with apex vessels will be robust and transferable between different manufacturers of apex vessels, as until now, no single proposed standard had been agreed.

The benefits of apex vessels are realized in three distinct scenarios:

1. A dissolution comparison, where, due to coning a difference was identified between batches or products in the standard vessel but not observed in an apex vessel and did not manifest in vivo.

2. When coning is observed, the first option is often to increase the paddle speed from $50 \mathrm{rpm}$ to 75 or higher (10) or move to the basket apparatus, which is not always effective in removing coning. By using apex vessels and removing coning, then it may be possible to reduce the paddle speed accessing a more discriminative method for non-artifacts, which would otherwise have been masked by increased paddle speed.
3. Apex vessels may represent a better enabler to evaluate formulation options. During development, changes in formulations are often required and dissolution is typically used to bridge between formulations as a way to build confidence that the new formulation will exhibit equivalent in vivo performance. This bridging can be severely hampered in the case where poor hydrodynamics in standard vessels leads to artifacts (e.g., coning), such that formulation developers may inadvertently limit compositional or processing options based on poor observed dissolution performance. For example, by limiting excipients, other critical quality attributes such as manufacturability or tensile strength may be less optimal due to the perceived need to avoid coning (in standard vessels).

The aim of this article is to provide case studies to describe the benefits of apex vessels and to report the results of an interlaboratory study across 11 partners, including five of the major dissolution bath manufacturers. Based on the results of the study, a standard specification and qualification procedure has been proposed for the use of apex vessels.

\section{METHODS AND MATERIALS FOR INTERLABORATORY STUDY \\ Participants}

Eleven partner organizations participated with each producing a dataset using their bath and vessel combination. All five major manufacturers of dissolution baths and their associated apex vessel were represented. Furthermore, each bath manufacturer design was tested a minimum of two times to allow an interlaboratory check on an individual vessel manufacturer, with the exception of the Hanson vessel, where a third party Quality Lab Accessories (QLA) vessel was used for partner number 7. Table 1 outlines the variations in baths at each participating laboratory. Partner number 1 also collected an $n=6$ reference profile using a standard vessel.

\section{Materials}

USP Prednisone Tablets RS (10 mg) were selected as the test article for the study due to their well-known propensity for coning and the familiarity of all partners in analysis methods due to their use in the USP performance verification test (PVT). A single batch, R080J0, was centrally purchased and distributed to all partners in sufficient quantity to conduct the test. Individual partners purchased the USP Prednisone RS with batch R083AO used by all participants. The protocol called for deaerated 
purified water; therefore, degassing was performed using the USP procedure or a validated equivalent, and if available, a dissolved oxygen meter used to confirm the level of not more than $6 \mathrm{ppm}$ (11) as stated in the USP toolkit 2.0.

Table 1. Outline of Bath and Vessel Details for Each Participant

\begin{tabular}{|c|c|c|}
\hline Partner No. & Bath Model & Vessel Manufacturer \\
\hline 1 & Agilent 708-DS & Agilent \\
\hline 2 & Distek 2500 Select & Distek \\
\hline 3 & Sotax ATXtend & Sotax \\
\hline 4 & Agilent 708-DS & Agilent \\
\hline 5 & Hanson Vision G2 Elite 8 & Hanson \\
\hline 6 & Distek 7100 & Distek \\
\hline 7 & Hanson Vision G2 Elite 8 & QLA \\
\hline 8 & Erweka DT820 & Erweka \\
\hline 9 & Sotax AT7 Smart & Sotax \\
\hline 10 & Sotax ATXtend & Sotax \\
\hline 11 & Erweka DT720 & Erweka \\
\hline
\end{tabular}

\section{Dissolution Bath Set-Up}

A USP Apparatus 2 bath was to be used and should ideally have been mechanically qualified in the 6 months prior to conducting the apex vessel study and the serial numbers of the exact apex vessels used were to be recorded. The distance between the top of the apex and the bottom of the paddle was to be set using a height gauge or other acceptable method to a target of $10.0 \mathrm{~mm}$ and be within the range of $9.0-11.0 \mathrm{~mm}$ where possible. The partner who used the Sotax AT7 Smart bath was not able to adjust height and so used a wider range (7-12 $\mathrm{mm})$. The centrality of the apex within the vessel was to be measured and recorded using digital calipers or an alternative, and the apex was ideally within $\pm 1 \mathrm{~mm}$ of the center of the vessel. The vessel internal diameter was also to be measured and recorded with digital calipers or an alternative. The reference profile was collected using a conventional paddle set-up.

\section{Dissolution Procedure}

A dissolution protocol was issued to each partner. Each partner conducted $n=12$ units in two separate $n=6$ dissolution runs using the same vessels and bath. Partner 9 conducted an $n=12$ dissolution using two baths and 12 individual vessels. A volume of $500 \mathrm{~mL}$ of deaerated purified water was dispensed gravimetrically and a paddle speed of $50 \mathrm{rpm}$ used for $30 \mathrm{~min}$ with time points taken at $5,10,15,20$, and 30 min prior to turning up to greater than $200 \mathrm{rpm}$ for an infinity spin time point at $45 \mathrm{~min}$. A $10-\mathrm{mL}$ sample was withdrawn at each time point and filtered with the first $5 \mathrm{~mL}$ discarded to waste. A $10-\mu \mathrm{m}$ ultrahigh molecular weight polyethylene cannula filter (Quality Lab Accessories [QLA], part\#FIL010-01-a, Telford, PA) followed by a Puradisc Whatman $0.45-\mu \mathrm{m}$ polyvinylidene difluoride (PVDF) filter (part \#67472504, Cytiva, Marlborough, MA) or a validated equivalent was used to filter the sample. A fresh syringe filter was specified for each time point, but the cannula filter could be reused. The samples were cooled to room temperature prior to analysis to ensure standard and sample were measured at an equivalent temperature or analyzed online by either fiber optic or flow-through ultraviolet (UV) spectrophotometry. If manual sampling was performed, then removed volume was corrected for in the calculation of percent dissolved. Careful observation of the dissolution runs was made with the position of tablet after addition to the bath and any unusual dissolution behavior was recorded. If automated sampling with flow-through online UV analysis was used, then validated filters must be used and a prime volume greater than $5 \mathrm{~mL}$ should be used. The prime volume is normally set to $1.5 \times$ the tubing volume of the sampling system, and the volume in vessels had to remain constant at $500 \mathrm{~mL}$ (i.e., no sample must be retained in the sample lines).

\section{Analytical Procedure}

UV spectrophotometry was used as the analysis technique to determine the concentration of prednisone dissolved from the Reference Standard tablet. The absorbance of the sample solution was compared to the absorbance of the Reference Standard material dissolved in dissolution medium. Two independent standard solution preparations were made, and the concordance was checked. The Reference Standard solution was prepared by dissolving $20 \mathrm{mg}$ in $20 \mathrm{~mL}$ of ethanol to make a standard stock solution and then diluting $10 \mathrm{~mL}$ of the standard stock solution and making up to $500 \mathrm{~mL}$ with dissolution medium to give a final concentration of $0.02 \mathrm{mg} / \mathrm{mL}$. UV measurements were performed at a wavelength of $242 \mathrm{~nm}$ and a path length of $10 \mathrm{~mm}$ using a quartz cell unless a fiber optic system was used. Concentrations measured were converted to percent dissolved accounting for changes in volume and sampling to form a dissolution profile.

\section{Profile and Statistical Analysis}

All participants submitted data associated with the experiment including vessel measurement details, standard details, filter details, visual observations, raw individual vessel data, and calculated data. Arithmetic 
means and percent relative standard deviations were then calculated for each partner. The $f_{2}$ calculation (12) was used to determine similarity between profiles.

Exploratory data analysis was carried out using Spotfire software (Tibco, Palo Alto, CA) to visualize raw data profiles, which is important to check for any outliers and to assess the distribution of data. A principal component analysis was carried out in SIMCA software (Sartorius, Goettingen, Germany) on the dissolution data to explore the similarity/dissimilarity between partners with respect to the mean dissolution profile. The factors captured by partners fall into two categories: a) those that are specific to the vessel, including internal diameter, apex deviation, and distance between apex and paddle; and b) general equipment descriptors, for example, the bath model. An analysis of covariance was carried out using JMP software (SAS, Cary, NC) to understand any potential impact of factors in category a. A one-way analysis of variance was applied to explore the potential influence of the factors in category b, including bath manufacturer and model as well as vessel manufacturer on dissolution.

\section{METHODS AND MATERIALS FOR CFD MODELING}

CFD was used to simulate the influence of small geometric differences in apex geometries of the five dissolution bath manufacturers on the hydrodynamics in the dissolution vessels. CFD can be used to quantify fluid-flow phenomena based on numerically solving the governing conservation laws of fluid transport. Simulations were performed using the CFD package Fluent (version 19.5.0, ANSYS Incorporated). For each geometry, an unstructured polyhedral hexcore mesh was generated. Inflation layers were created around the paddle blade, shaft, and vessel wall to capture near-wall behavior. A sliding mesh interface was created, separating the paddle blade and shaft from the remainder of the vessel to allow for rotation. The meshes typically contained between 350,000 and 360,000 cells. In all simulations, the vessel, paddle, and shaft walls were defined using no-slip boundary conditions. The air-water interface was assumed to be flat and was defined as a symmetry boundary condition to allow slip.

The flow in the vessels is expected to be turbulent, and therefore, the choice of turbulence model is critical for generating simulations close to physical reality. Based on previous simulations and comparison with experimental measurements, the $k-\omega$ turbulence model with low Reynolds number correction was selected $(4,13)$. Transient simulations were solved until results reached a pseudo-steady state. Results were extracted by compiling data from a full revolution. Simulations were conducted at a paddle speed of $50 \mathrm{rpm}$. Fluid properties were defined to model pure water at $37^{\circ}$, with a density of $993 \mathrm{~kg} / \mathrm{m}^{3}$ and a viscosity of $6.92 \times 10^{-4} \mathrm{~Pa} \cdot \mathrm{s}$.

\section{CASE STUDIES}

The following six case studies all demonstrate the different benefits that a broader acceptance of apex vessels can bring.

\section{Case Study 1}

Two different enteric-coated delayed release pellet formulations ( $A$ and $B$ ) containing the same active pharmaceutical ingredient (API) were tested in USP Apparatus 2 using both the standard vessel and the original VanKel apex vessel at $100 \mathrm{rpm}$. Formulation $A$ had a pellet diameter of $0.4 \mathrm{~mm}$ and a density of $1.4 \mathrm{~g} / \mathrm{cm}^{3}$, and formulation $B$ had a pellet diameter of $0.6 \mathrm{~mm}$ and $a$ density of $1.3 \mathrm{~g} / \mathrm{cm}^{3}$. Both formulations were compared in the buffer stage of the USP delayed release method using $1 \mathrm{~L}$ of $\mathrm{pH} 6.8$ phosphate buffer and drug release quantified by high-performance liquid chromatography (HPLC). In addition, both formulations were tested in a bioequivalence study using a single dose, crossover design in 18 healthy subjects.

Coning of the pellets was observed in the standard vessel even at $100 \mathrm{rpm}$, which also manifested itself as high variability, when the same formulation was tested in an apex vessel variability was greatly improved (Figure 1). The standard vessel (Figure 2) also predicted a difference between formulations, but profiles were observed to be more similar in the apex vessel (Figure 3). This was confirmed by the in vivo data in healthy volunteers, which showed the standard vessel was identifying an in vitro artifact of a greater propensity to cone related to a higher density and smaller pellet size that was of no in vivo relevance (Table 2). This indicates that when visual coning is observed, it is important to assess any differences when the in vitro artifact is removed. In this example, it may also have been prudent to reduce the paddle speed in the apex vessel to ascertain if any discrimination was being masked using the 100-rpm paddle speed.

\section{Case Study 2}

A weakly basic drug substance was formulated as a roller compacted tablet containing both microcrystalline cellulose and dicalcium phosphate anhydrous as fillers. The solubility and permeability of the drug substance was measured and shown to be tentatively assigned as a high solubility, high permeability drug substance with a minimum solubility of $2 \mathrm{mg} / \mathrm{mL}$ at $\mathrm{pH} 6.8$. This would allow 


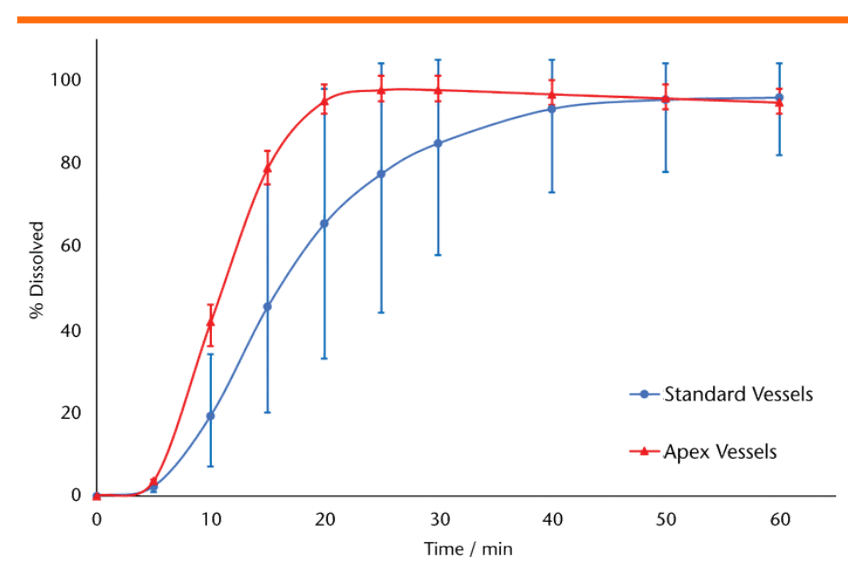

Figure 1. Formulation A in standard vessels vs. apex vessels at $100 \mathrm{rpm}$ ( $n=6$; error bars represent maximum and minimum values).
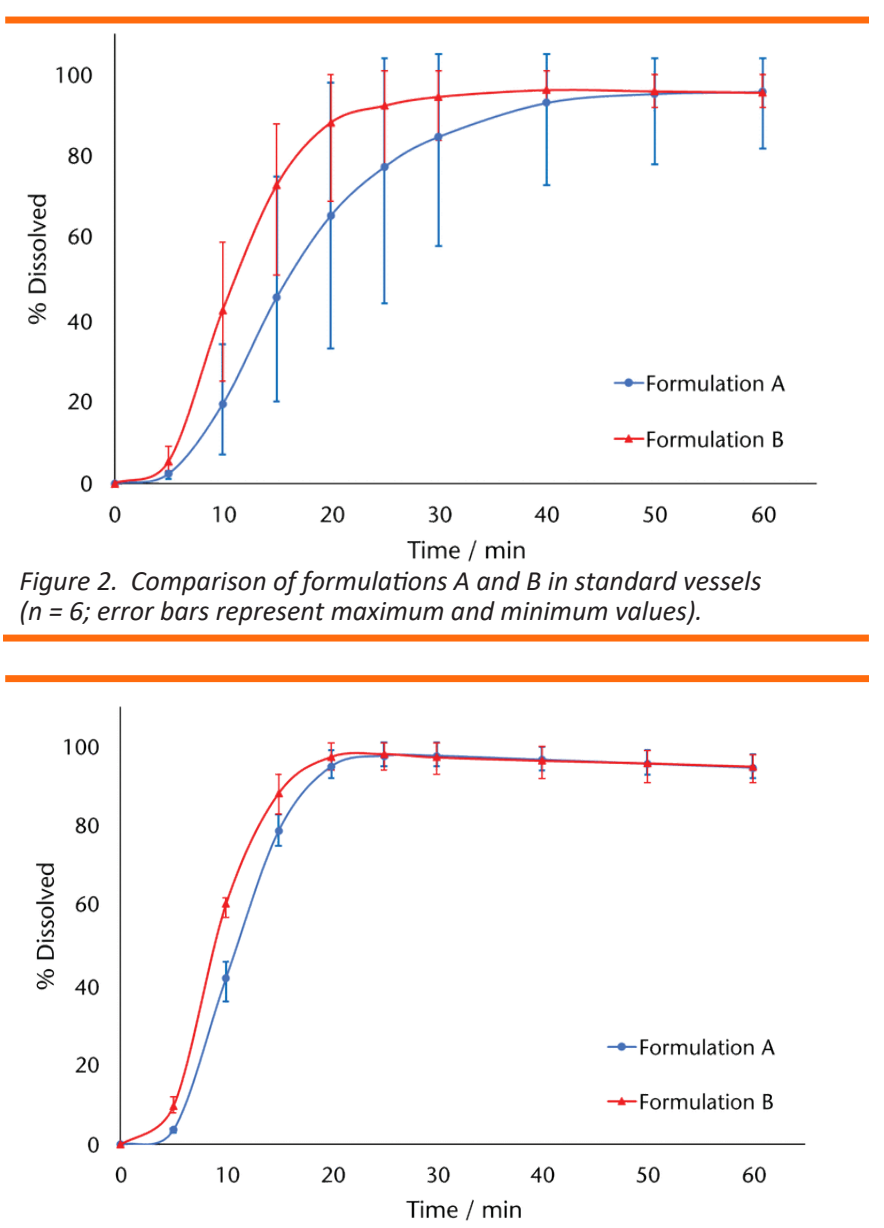

Figure 3. Comparison of formulations $A$ and $B$ in apex vessels ( $n=6$; error bars represent maximum and minimum values).

Table 2. Bioequivalence Study: Comparison of Geometric Mean Ratios and 95\% Confidence Intervals of Formulations $A / B(n=18)$

\begin{tabular}{|c|c|c|}
\hline & Geometric Mean Ratio & 95\% Confidence Interval \\
\hline Cmax ratio (A/B) & 1.18 & $0.96-1.45$ \\
\hline AUC ratio (A/B) & 1.10 & $0.98-1.23$ \\
\hline $\operatorname{Tmax}(\mathrm{h})$ & $\begin{array}{c}\text { Formulation A }=2.0 ; \\
\text { Formulation } B=2.1\end{array}$ & - \\
\hline
\end{tabular}

$A \cup C=$ area under the curve. access to BCS 1 biowaivers as described in International Council for Harmonisation guideline M9 (ICH M9) if the in vitro dissolution can meet the requirements of $\geq 85 \%$ dissolved in $\leq 30 \mathrm{~min}$. The dissolution conditions specified in ICH M9 (14) are $900 \mathrm{~mL}$ or less of medium, $50 \mathrm{rpm}$ using the paddle apparatus or $100 \mathrm{rpm}$ using the basket apparatus, tested in three different media: $\mathrm{pH} 1.2,4.5$, and 6.8 pharmacopoeial buffers. It does state that when high variability is observed in the paddle apparatus, the use of baskets at $100 \mathrm{rpm}$ is recommended, and alternative methods such as the use of sinkers or other appropriately justified approaches may be considered to overcome issues such as coning, if scientifically substantiated. It is the authors' experience that sinkers do not improve coning for tablets or granules, only the act of increasing the paddle speed or switching to an apex vessel will improve the situation. For some formulations, the switch to the basket apparatus can improve the situation but is not a universal solution. The issue is that moving away from the standard hydrodynamic conditions, the global acceptability gained from the ICH M9 guideline becomes more uncertain and carries a greater regulatory risk.

In this example, the BCS 1 acceptance criterion could be met at $\mathrm{pH} 1.2$ and 4.5 using the specified hydrodynamic conditions but at $\mathrm{pH} 6.8$, this was not possible due to the increased impact of the coning on the extent of dissolution. Figure 4 demonstrates that when using a paddle apparatus at $50 \mathrm{rpm}$ or the basket apparatus at $100 \mathrm{rpm}$, only approximately $60 \%$ and $70 \%$ was dissolved at $30 \mathrm{~min}$, respectively. This increases to a borderline pass at $85 \%$ at a paddle speed of $75 \mathrm{rpm}$, and in apex vessels at a 50 -rpm paddle speed, it further increases to $90 \%$. Coning is evident in all scenarios as evidenced by the steep increase seen post $60 \mathrm{~min}$ when the paddle speed was increased to $200 \mathrm{rpm}$ for the infinity spin. Furthermore, a cone on the apex is visibly present in the apex vessels at $50 \mathrm{rpm}$ (Figure 5), which indicates the design of an apex vessel is a vast improvement, but with high levels of dense insoluble excipients, coning is still possible.

\section{Case Study 3}

A situation arose where an early Phase 1 and 2 tablet formulation was found to be unsuitable for commercialization such that further tablet development was necessary prior to the start of the pivotal study. Criteria for a successful formulation switch were developed and included manufacturability attributes such as flow, compaction properties, friability, picking/ sticking, acceptable process margins, and speed sensitivity. This was balanced against the need for in vitro bridging to avoid a relative bioavailability study and to 
show equivalence and build internal confidence in the pivotal formulation, due to the cost and timeline delay that would be incurred. The early formulation had a fairly rapid and complete release in USP Apparatus 2 at $50 \mathrm{rpm}$ across the three media spanning the physiological range and contained no excipients that caused coning. When the improved formulation development started and focused on manufacturability, the inclusion of high levels of insoluble excipients such as microcrystalline cellulose and dicalcium phosphate anhydrous resulted in severe coning that, under standard hydrodynamic conditions, could not be removed. In the case of formulations N3 and $\mathrm{N} 4$, they were not even equivalent in an apex vessel and failed to meet the dissolution target defined in the quality target product profile suggesting a bridging risk. The formulators attempted to explore different combinations of fillers and disintegrants and found that many (formulations N7-N10) were not robust with respect to manufacturability, but did point to a solution (N11) that would meet all criteria with respect to the in vitro bridge and manufacturability. Table 3 shows the red/amber/green risk (RAG) analysis conducted on the formulation options, demonstrating the balance between manufacturability and the ability to achieve an in vitro bridge to the formulation used in early studies. Formulations N5 and N6 were flagged as amber risks for achieving this in vitro bridge under standard apparatus and conditions and green for all manufacturability elements evaluated. The amber in vitro risk was due to the inability to show similarity under standard hydrodynamic conditions. Although using an apex vessel to remove the coning both formulations N5 and N6 showed similarity. It was noted that had apex vessels been globally acceptable, the formulation development would have been faster, as development would likely have focused in on the N5 and N6 variants, instead of with development continuing to the N11 variant. It would also have been less resourceconsuming and with very little in vivo risk because the mechanism of the observed slowdown was related to the dense insoluble excipients used to improve the manufacturability.

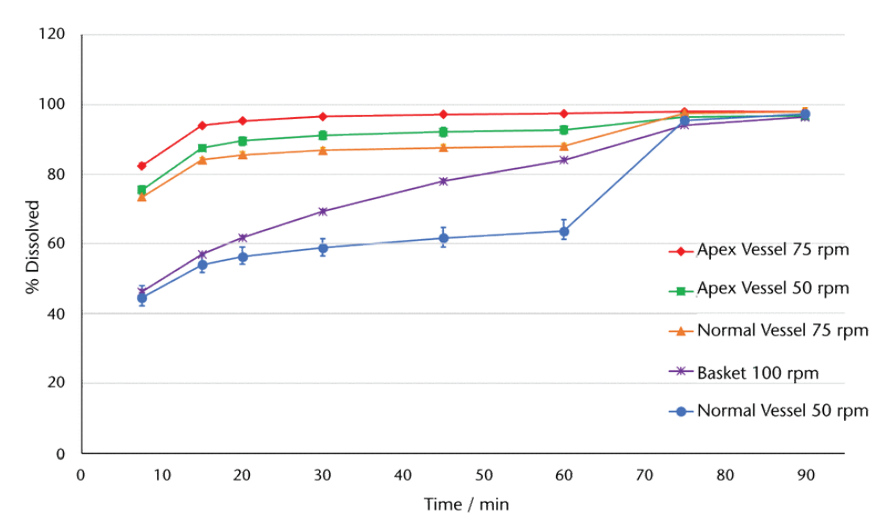

Figure 4. Comparison of hydrodynamic conditions for BCS 1 drug substance in tablet with insoluble dense filler at $\mathrm{pH} 6.8$ ( $n=3$ per condition; error bars represent maximum and minimum values).

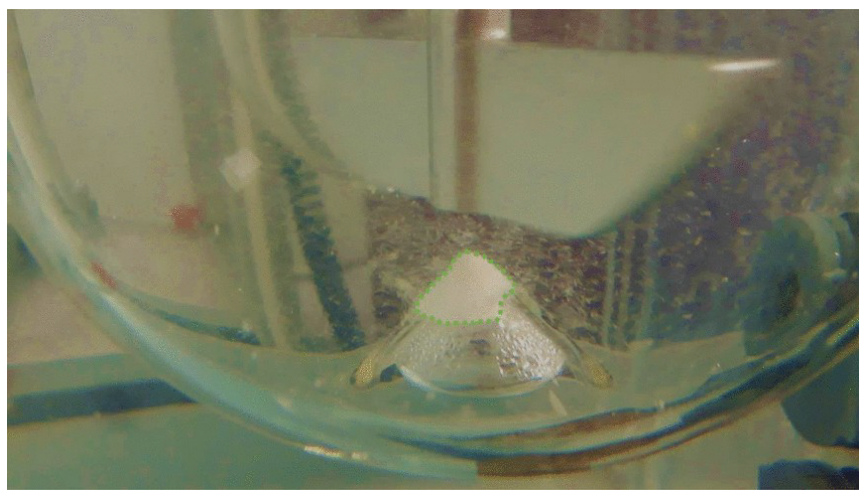

Figure 5. Evidence of coning in an apex vessel at $50 \mathrm{rpm}$.

Table 3. RAG Analysis of Formulation Options Demonstrating Trade-Off Between in Vitro Bridge and Manufacturability

\begin{tabular}{|c|c|c|c|c|c|c|c|c|c|c|}
\hline Batch & N2 & N3 & N4 & N5 & N6 & N7 & N8 & N9 & N10 & N11 \\
\hline Flow & & & & & & & & & & \\
\hline Compaction & & & & & & & - & - & - & High Risk \\
\hline Friability & & & & & & & & & & \\
\hline Disintegration & & & & & & & & & & \\
\hline Picking/Sticking & & & & & & & & & & \\
\hline In Vitro Bridge & & & & $*$ & $*$ & & & & & \\
\hline to mitigate & No risk option \\
\hline In vivo concern due to high quality of mannitol & & & & & & & & & & \\
\hline Process Margin & - & - & - & - & - & & & & & \\
\hline Speed Sensitivity & - & - & - & - & - & & & & & \\
\hline Chemical Stability & - & - & - & & & & - & - & - & \\
\hline Dissolution Stability & - & - & - & - & - & & - & - & - & \\
\hline
\end{tabular}

(-, was not evaluated fully; *, if apex vessels had been accepted development would have focused on N5 and N6 and not continued to new variants. 


\section{Case Study 4}

Dissolution methodologies that provide a non-sink environment with appropriate hydrodynamics are valuable tools for assessing a formulation's ability to generate and maintain supersaturation, a critical success factor in the development of amorphous solid dispersion (ASD) formulations of poorly water-soluble molecules. They also have the potential to serve as a predictive tool (clinical relevance) for predicting in vivo performance and product quality.

Most ASD-based formulations contain a polymer such as copovidone or hydroxypropyl methylcellulose acetate succinate. These polymers, especially copovidone when used in high quantities, can render slow erosion in tablets and slow drug release in conventional dissolution apparatuses (USP Apparatus 1 or 2). This is often due to the insufficient hydrodynamics and mechanical forces within conventional dissolution vessels.

Compound $\mathrm{X}$ is an ionizable compound with two pKa values. The drug substance in a crystal form exhibits very poor aqueous solubility. Therefore, the drug product was prepared as an ASD in a polymer-surfactant matrix to improve the product's bioavailability. This case study aimed to develop a clinically relevant non-sink dissolution method using simple buffer solutions for several prototype ASD formulations with various drug loadings.

Three method conditions were found to be critical in achieving clinical relevance: surfactant level (zero surfactants), strong hydrodynamics, and the use of dual pHs. A dissolution method was developed utilizing a twostage dual $\mathrm{pH}$ medium and apex vessels containing no surfactant with adequate hydrodynamics. As drug-rich species formed in ASD dissolution are often overlooked, filter pore size also played a role in achieving the desired outcome. Among the three critical method conditions, adequate hydrodynamics was the key to achieving a good correlation between the in vitro release rate and the deconvoluted pharmacokinetic (PK) results. To demonstrate the impact on dissolution caused by varying hydrodynamics, the same method conditions were applied with compendial vessels and apex vessels, respectively: USP Apparatus 2 at $125 \mathrm{rpm}, 500 \mathrm{~mL}$ of $0.1 \mathrm{~N}$ hydrochloric acid for $15 \mathrm{~min}$, followed by $400 \mathrm{~mL}$ of $0.113 \mathrm{M}$ phosphate buffer. As shown in Figure 6 and Figure 7, the release rate of the reference (commercial) formulation was significantly underestimated using compendial vessels. Although the rank order of these three formulations is aligned with the deconvoluted PK results shown in Figure 8, the fast and complete release profile of the $20 \%$ drug load hot-melt extrusion (HME) formulation (20\% HME) compared to the commercial formulation could easily mislead the formulation decision. These results also suggest the dissolution testing using compendial vessels overly discriminated against the drug load changes made in the prototype formulation. In contrast, the use of the same dissolution conditions with the substitution of the apex vessels provided a much closer relationship between the in vitro and in vivo results.

This method should provide a robust correlation with in vivo performance of the two prototype ASD formulations. It was also used to provide a fundamental understanding of in vivo performance, especially as the in vivo results were not as expected. The correlation between dosenormalized $\mathrm{C}_{\max }$ and in vitro percent of released obtained from commercial, $20 \% \mathrm{HME}$, and $25 \% \mathrm{HME}$ formulations at multiple time points were assessed internally and rendered positive results. Its credibility was further validated as it successfully predicted the bioequivalence of the proposed Compound $\mathrm{X}$ formulations with higher strengths.

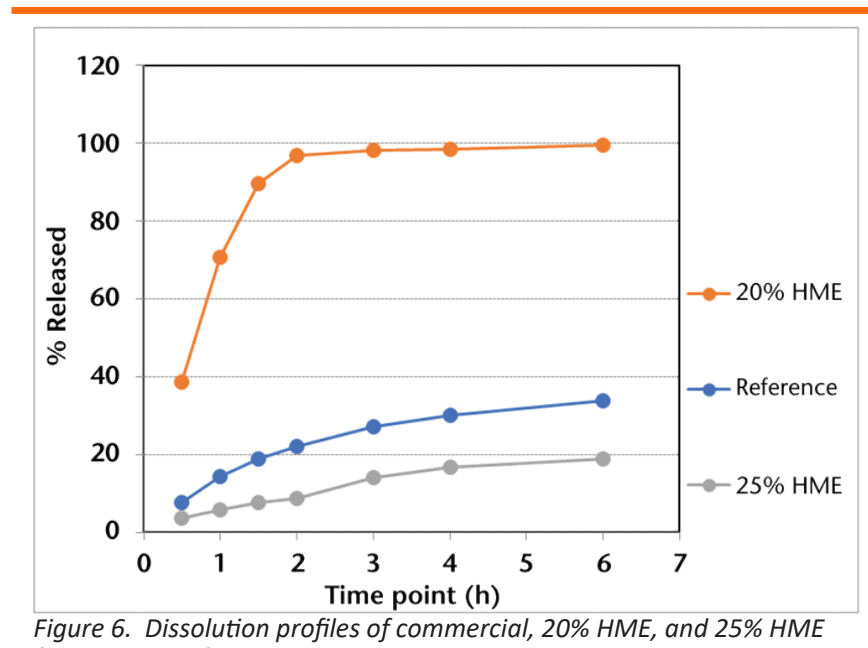

Figure 6. Dissolution profiles of commercial, 20\% HME and 25\% HME formulations of Compound $X$ obtained using compendial vessels.

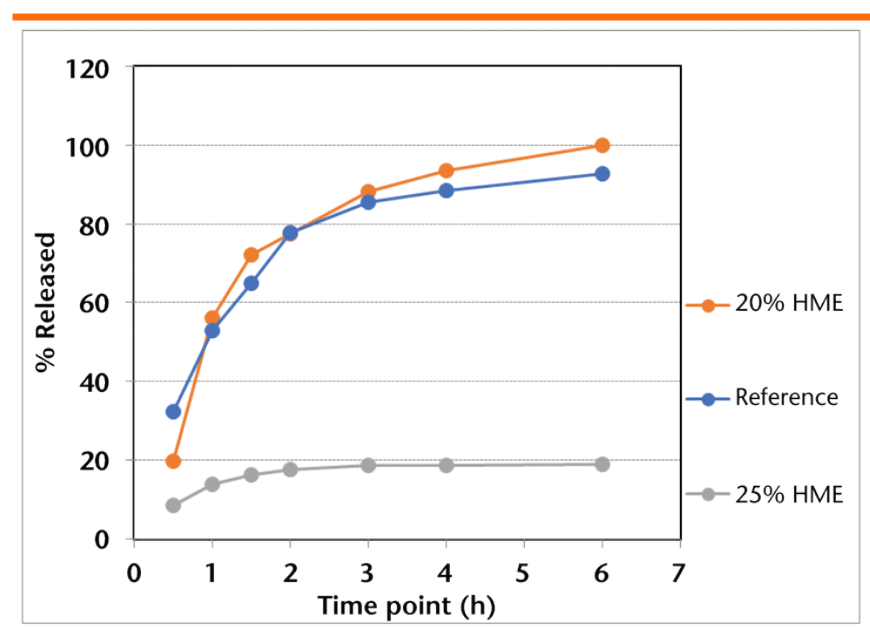

Figure 7. Dissolution profiles of commercial, 20\% HME, and 25\% HME formulations of Compound $X$ obtained using apex vessels. 


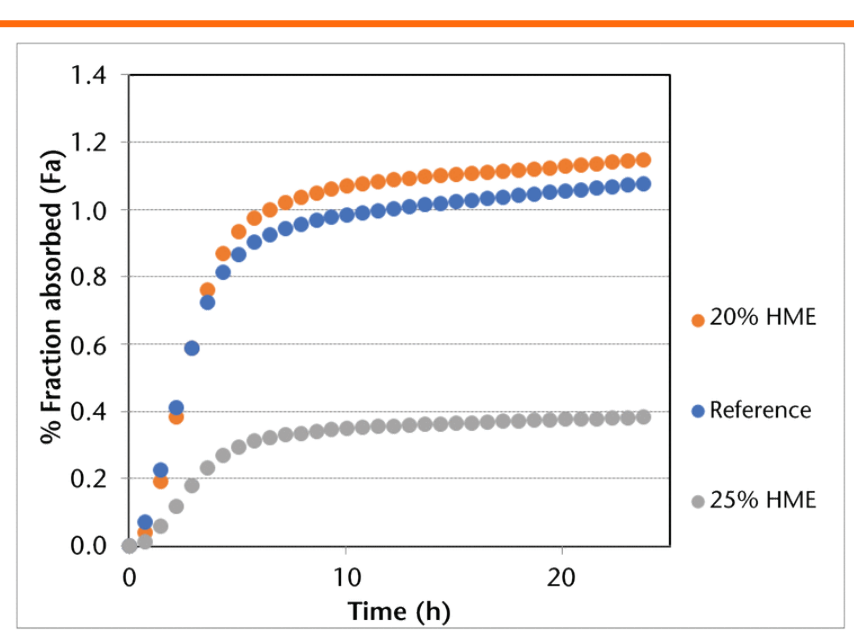

Figure 8. Deconvoluted PK results of commercial, 20\% HME, and 25\% HME formulations of Compound $X$.

\section{Case Study 5}

A weakly basic drug was formulated as an immediate release tablet with a drug load of approximately $30 \%$ and included the water- insoluble excipients anhydrous dibasic calcium phosphate and microcrystalline cellulose. Dissolution method development was performed using the nominal formulation and two variant tablet formulations. One variant formulation consisted of a drug substance with a larger particle size than the target. The second variant formulation, referred to as a "slow variant", was a composite variant that contained a lower level of disintegrant and higher level of lubricant to achieve a tablet with a significantly slower dissolution profile.

The drug is highly soluble in $0.1 \mathrm{~N}$ hydrochloric acid whereas its solubility at $\mathrm{pH}$ values of 4.5 and higher is less than $3 \times$ sink conditions. Figure 9 shows insufficient sink conditions for the nominal formulation in $\mathrm{pH} 4.5$ and $\mathrm{pH}$ 6.8 media, as $100 \%$ drug dissolution is not achieved. In
$0.1 \mathrm{~N}$ hydrochloric acid, tablets were observed to cone when using paddles at $50 \mathrm{rpm}$. At $75 \mathrm{rpm}$, no coning is observed, but there is no discrimination under this condition between the nominal formulation and the large particle size variant and very limited discrimination between the nominal formulation and the slow variant.

A dissolution medium at $\mathrm{pH} 3.5$, using $65 \mathrm{mM}$ citratephosphate buffer, which is intermediate between $0.1 \mathrm{~N}$ hydrochloric acid and $\mathrm{pH} 4.5$, was evaluated. As shown in Figure 10, coning was observed with a paddle speed of $50 \mathrm{rpm}$ for the nominal formulation. At $75 \mathrm{rpm}$, no coning is observed in the profile and there is discrimination between the nominal formulation and two variants. Although acceptable discrimination was achieved at $\mathrm{pH} 3.5$ and $75 \mathrm{rpm}$, this condition does not result in a rugged method, as different profiles are observed with different instrument types (semi-automated system and vessels from one vendor and a fully automated system and vessels from another vendor) as shown in Figure 11. Coning was observed for one instrument type but not the other for an accelerated stability sample [6 months at $40^{\circ} / 75 \%$ relative humidity $\left.(\mathrm{RH})\right]$ of the nominal formulation and the slow variant formulation. For the samples where no coning is observed in the profile, there is mass observed in the bottom of the vessel. Coning with one instrument type but not the other may be the result of slight but significant differences in hydrodynamics or vibration between the two systems.

The use of apex vessels to eliminate coning and the impact of mass at the bottom of the vessel and to potentially improve method ruggedness was evaluated. As shown in Figure 12, paddles with apex vessels at $50 \mathrm{rpm}, \mathrm{pH} 3.5$ do not show a coning effect and dissolution profiles between

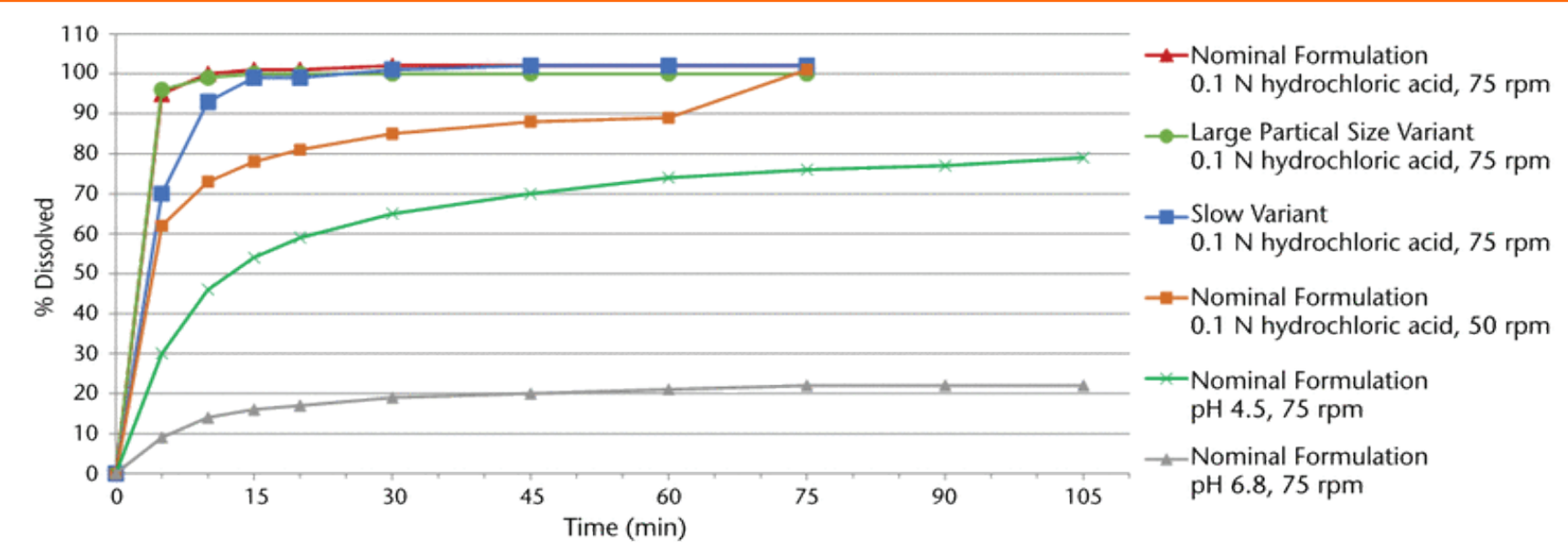

Figure 9. Mean dissolution profiles using paddles with standard vessels for the nominal formulation in $0.1 \mathrm{~N}$ hydrochloric acid at $50 \mathrm{rpm}(\mathrm{n}=3$ ); nominal formulation $(n=6)$, large particle size variant $(n=2)$, and slow variant $(n=6)$ in $0.1 \mathrm{~N}$ hydrochloric acid at $75 \mathrm{rpm}$; nominal formulation $(n=12)$ in pH 4.5 and 6.8 at $75 \mathrm{rpm}$. All profiles have an infinity spin for the last $15 \mathrm{~min}$. 


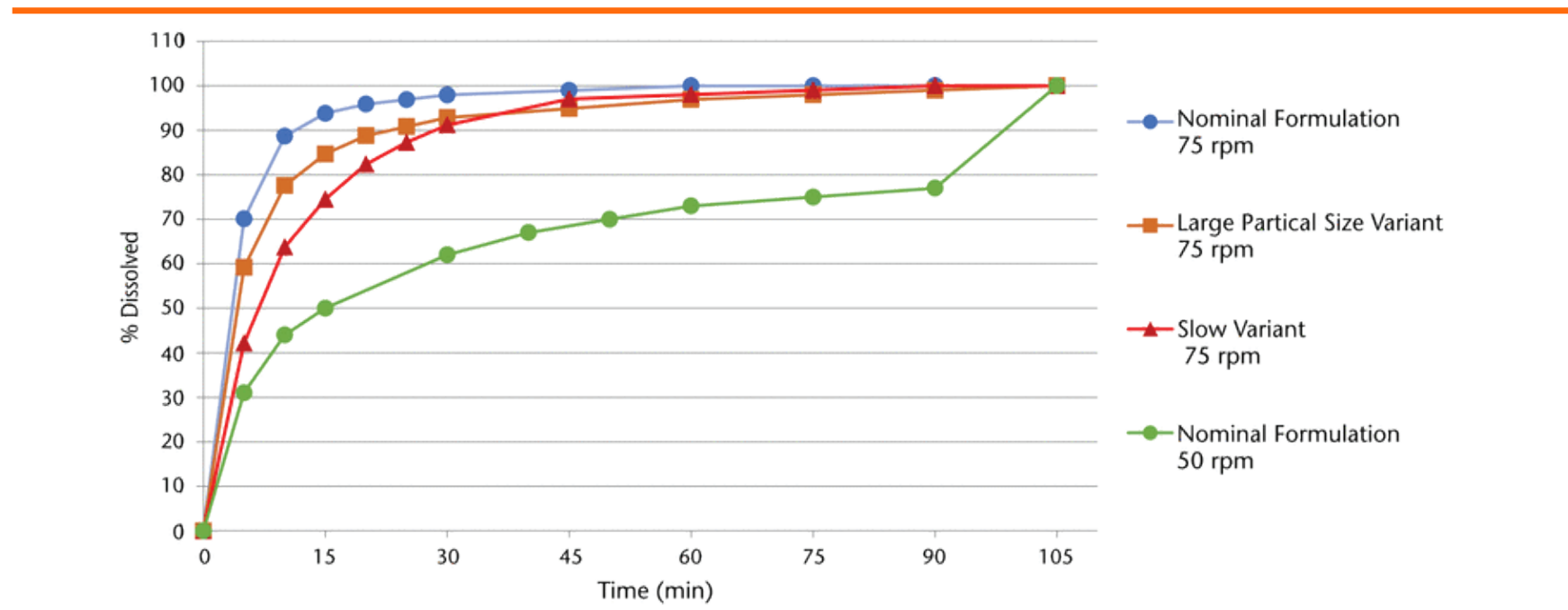

Figure 10. Mean dissolution profiles using paddles with standard vessels, $\mathrm{pH} 3.5$ for the nominal formulation at $50 \mathrm{rpm}(\mathrm{n}=3)$ and the nominal formulation $(n=3)$, large particle size variant $(n=3)$, and slow variant $(n=12)$ at $75 \mathrm{rpm}$.
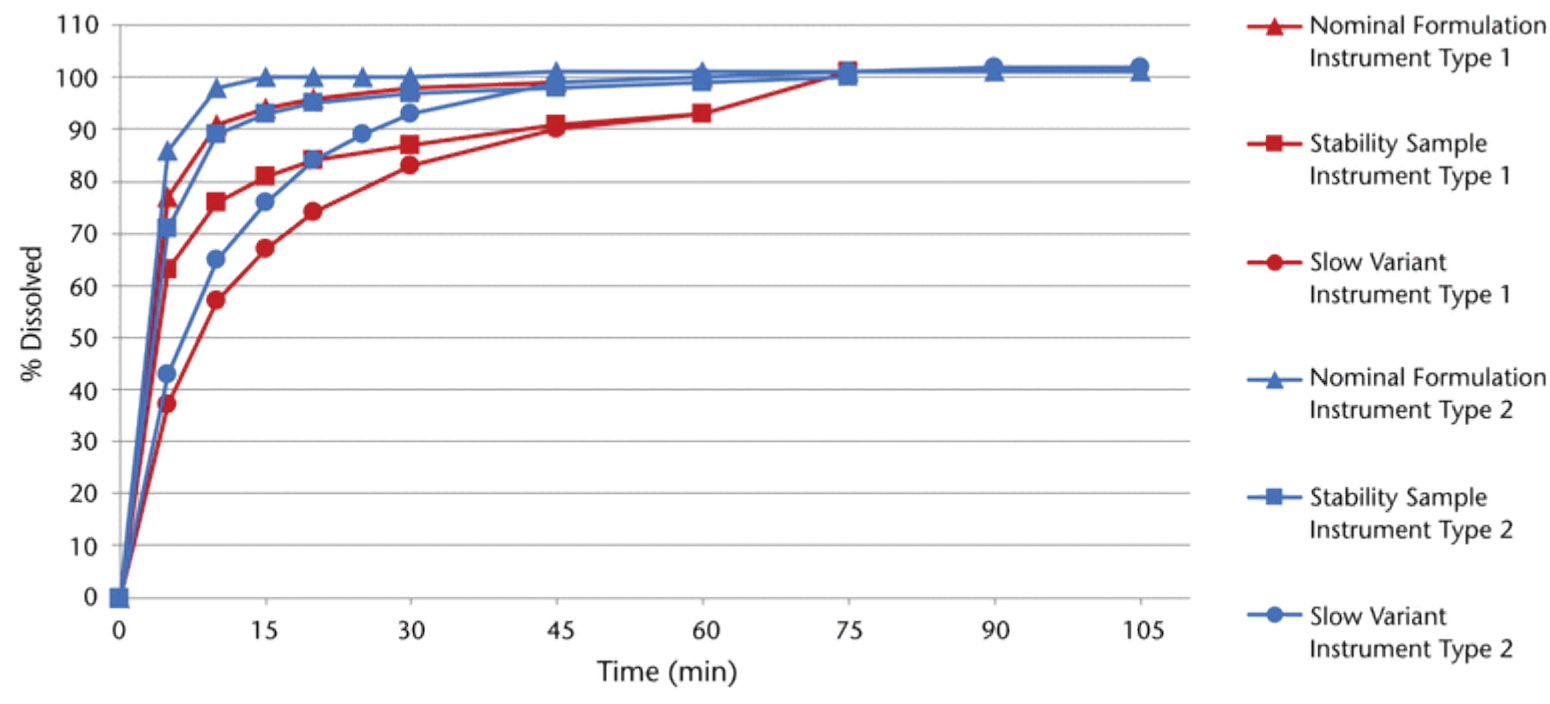

Red profiles indicate instrument manufacturer 1 and a semiautomated system. Blue profiles indicate instrument manufacturer 2 and a fully automated system.

Figure 11. Mean dissolution profiles ( $n=3,6$, or 12) using paddles, $p H 3.5$ with standard vessels at 75 rpm for various samples with different instrument types.

the two instrument types are similar, showing $>85 \%$ in 15 min or meeting $f_{2}\left(f_{2}=54\right.$ for the slow variant). This demonstrates that the improved hydrodynamics of the apex vessels are helpful in minimizing this type of artifact.

A method robustness design of experiments (DOE) study was conducted to evaluate the apex vessel method at $\mathrm{pH}$ 3.5 to determine whether there were any sensitivities in the method conditions. An initial dissolution robustness study (22-run half-fractional factorial design with 6 center points), which evaluated the effect of $\mathrm{pH}$, agitation speed, medium volume, and medium temperature on dissolution, indicated that $\mathrm{pH}$ and agitation speed were the two factors that had a significant impact on dissolution. Based on this knowledge, a follow-up dissolution robustness DOE study using a two-factor $3 \times 3$ full factorial design was used to study the effect of tablet type [control versus stressed (exposed at $50 \% 75 \%$ RH for 2 weeks)], medium $\mathrm{pH}$, and agitation speed on dissolution. By incorporating the original DOE runs into the follow-up $D O E$, the total number of runs included in the robustness DOE analysis are 19 for each of the tablet types. Based on the robustness assessment, paddles with apex vessels at $55 \mathrm{rpm}$ and $\mathrm{pH} 3.5$ was selected as the final dissolution method. Dissolution profiles with this method are shown in Figure 13 and demonstrate method discrimination and instrument-to-instrument ruggedness. 

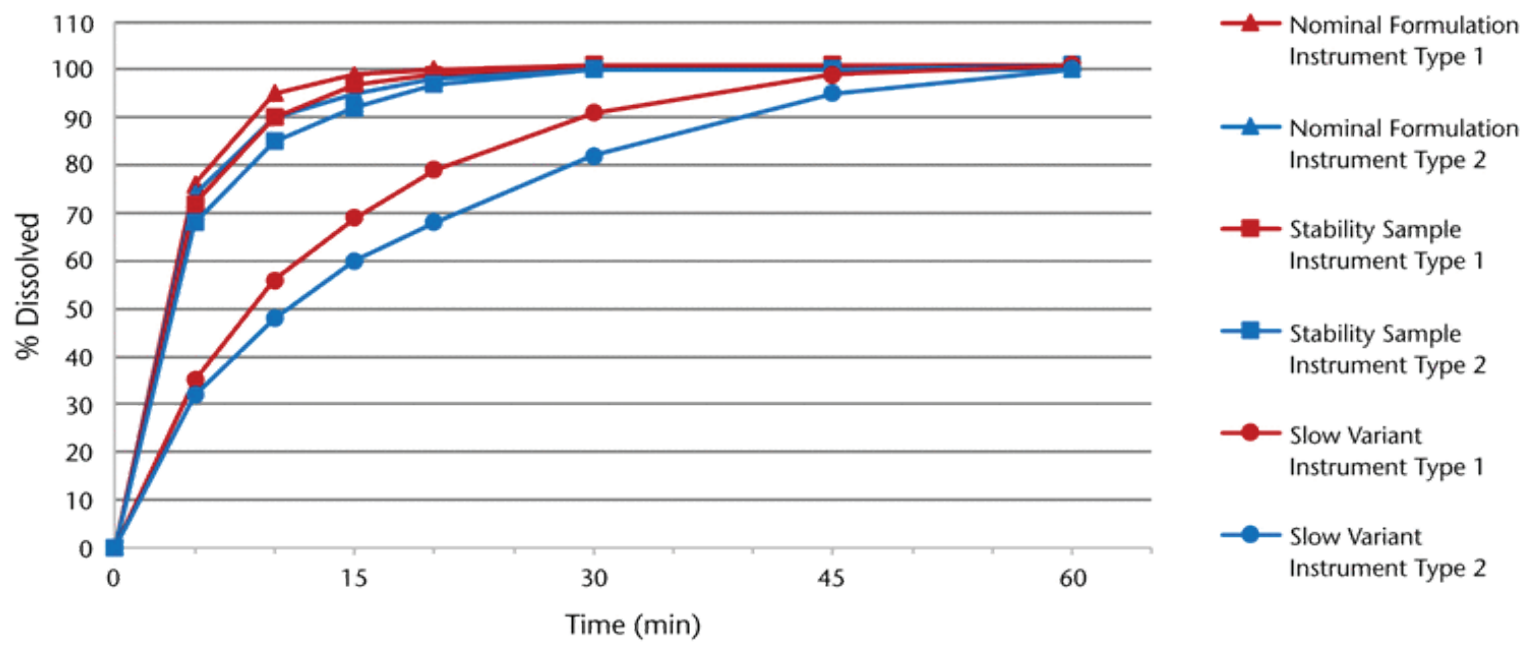

Red profiles indicate instrument manufacturer 1 and a semiautomated system. Blue profiles indicate instrument manufacturer 2 and a fully automated system.

Figure 12. Mean dissolution profiles ( $n=3,6$, or 12) using paddles, $p H 3.5$ with apex vessels at 50 rpm for various samples with different instrument types.

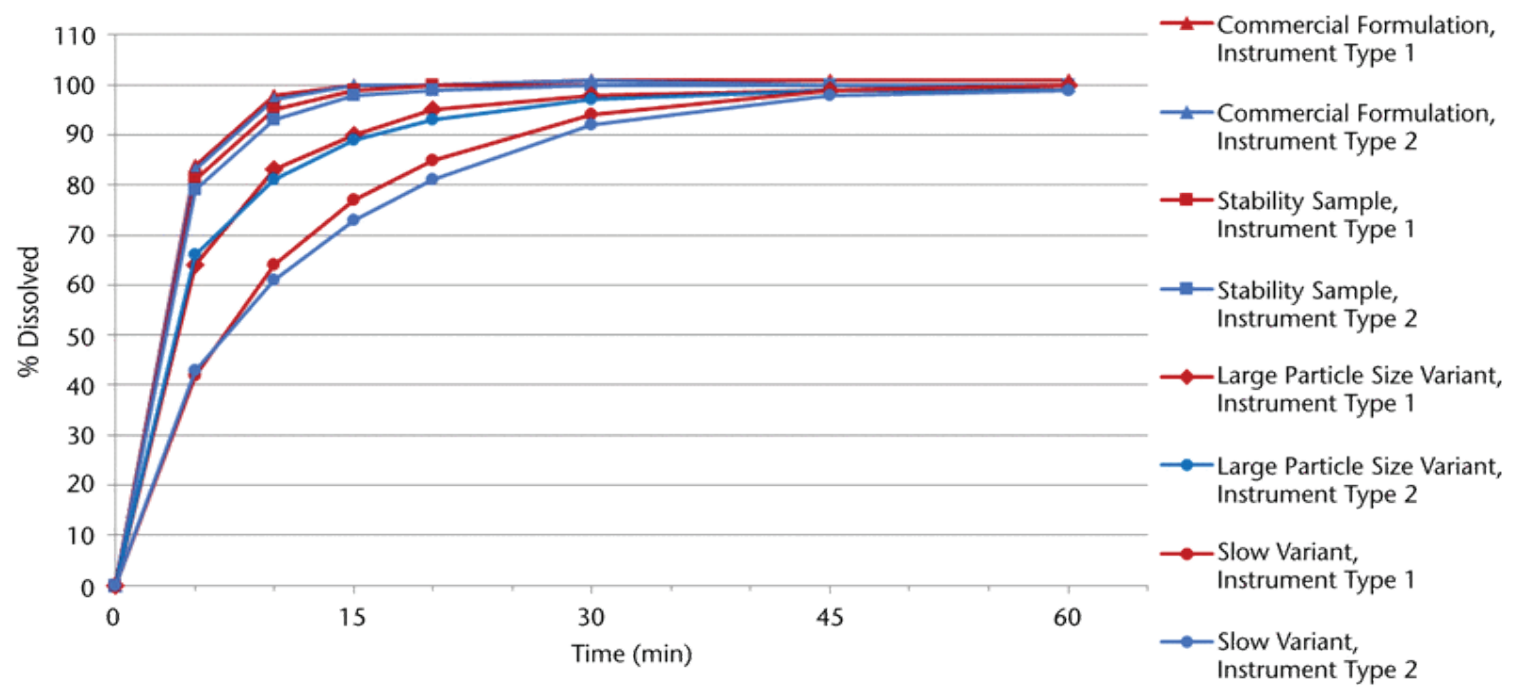

Red profiles indicate instrument manufacturer 1 and a semiautomated system.

Blue profiles indicate instrument manufacturer 2 and a fully automated system.

Figure 13. Mean dissolution profiles $(n=6)$ using paddles with apex vessels, $55 \mathrm{rpm}, \mathrm{pH} 3.5$ demonstrating discrimination and robustness between instrument types.

The use of paddles with apex vessels enabled the development of a discriminating, robust, and rugged method for an immediate release tablet formulation. This could not have been achieved for this product with standard vessels because of the need to balance method discrimination and method ruggedness. Whereas method discrimination was achieved with standard vessels at $\mathrm{pH}$ 3.5 and $75 \mathrm{rpm}$, this condition was not rugged as different profiles were observed between instrument types due to slight but significant differences in hydrodynamics. Conversely, method ruggedness was achievable with standard vessels using $0.1 \mathrm{~N}$ hydrochloric acid and $75 \mathrm{rpm}$, but this condition provided inadequate discrimination. The use of apex vessels at $\mathrm{pH} 3.5$ and $55 \mathrm{rpm}$ provides a discriminating, robust, and rugged method for this product.

\section{Case Study 6}

This case involved dissolution using apex vessels in biorelevant media for a biopharmaceutics risk assessment of a formulation change in early development. When a tablet formulation was developed for a Phase 2 study, 
several changes were made from the original Phase 1 formulation, including excipient types and API particle size, to improve processing ability. The Phase 1 tablets are $1 \mathrm{mg}$ and $10 \mathrm{mg}$ using a common granulation. The Phase 2 tablets are $2 \mathrm{mg}$ and $10 \mathrm{mg}$ using a granulation that is different from Phase 1. A biopharmaceutics assessment was conducted, including in vitro, in silico, and in vivo dog studies, to evaluate whether the performance of the two tablet formulations are similar. During the in vitro study in biorelevant media, both the 10-mg Phase 1 and Phase 2 tablets and the 2-mg Phase 2 tablet showed coning when normal vessels were used, causing slow dissolution and high variability. The 2-mg Phase 2 tablet behaved very differently from the $2 \times 1-\mathrm{mg}$ Phase 1 tablet. However, when the normal vessels were replaced with apex vessels, the coning problem was resolved for all tablets. Figure 14 shows that the dissolution profiles generated using both normal vessels and apex vessels for the $2 \times 1$-mg Phase 1 tablet are very similar but very different for the 2 -mg Phase 2 tablet. In Figure 15, the coning is observed for both $10-\mathrm{mg}$ tablets with the same API particle size $\left(D_{90}\right.$ $=23 \mu \mathrm{m}$ ) when normal vessels were used. For the tablet with a larger particle size of API $\left(D_{90}=50 \mu \mathrm{m}\right)$, the coning was more severe. However, when apex vessels were used, all 10-mg tablets showed fast drug release despite the API particle size and regardless of which formulation.

The comparability of the tablets was further assessed using a human physiologically based pharmacokinetic (PBPK) model in GastroPlus based on first-in-human data. $Z$ factors were fitted to the in vitro biorelevant dissolution data from the apex vessel. Simulation results predicted that no significant difference at 2-mg and $10-\mathrm{mg}$ dose strengths, as indicated by a $<1 \%$ change in fraction absorbed observed between the Phase 1 tablets $(23 \mu \mathrm{m}$ $\mathrm{API}$ ) and Phase 2 tablets (50 $\mu \mathrm{m} \mathrm{API).}$

An in vivo dog study was also performed to assess the equivalence of the tablets, including 8 dogs divided into two groups for crossover of 2-mg dose and 10-mg dose, respectively. The dog PK data (Figure 16) indicated that the 2-mg Phase 2 tablet is equivalent to the $2 \times 1-\mathrm{mg}$ Phase 1 tablet. The 10-mg Phase 2 tablet showed a 10\% decrease in mean relative bioavailability compared with the 10-mg Phase 1 tablet, primarily attributed to one dog that showed unique behavior when compared with others within the group.

Based overall on the in vitro, in silico, and in vivo assessment, it was concluded that the 2-mg and $10-\mathrm{mg}$ Phase 2 tablets are expected to perform similarly to the 2x 1-mg and 10-mg Phase 1 tablet dosage forms. The in vitro performance using the apex vessels correlated well with the predictions from in silico and in vivo studies.

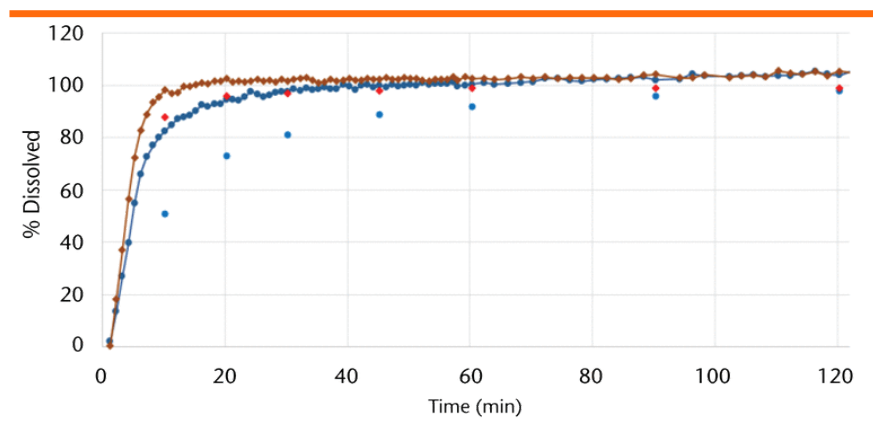

$\rightarrow$ Phase 2, 2-mg AV $\rightarrow$ Phase 1, 2x 1-mg AV $\bullet$ Phase 2, 2-mg NV - Phase 1, 2× 1-mg NV Figure 14. Mean dissolution profiles $(n=3)$, Phase 2 tablets $(2 \mathrm{mg}, 23 \mu \mathrm{m}$ D90) vs. Phase 1 tablets $(2 \times 1 \mathrm{mg}, 23 \mu \mathrm{m}$ D90) in Fasted State Simulated Intestinal Media-version 1 (FaSSIF-V1), 500 mL, 50 rpm, normal vessel (NV) vs. apex vessel (AV).
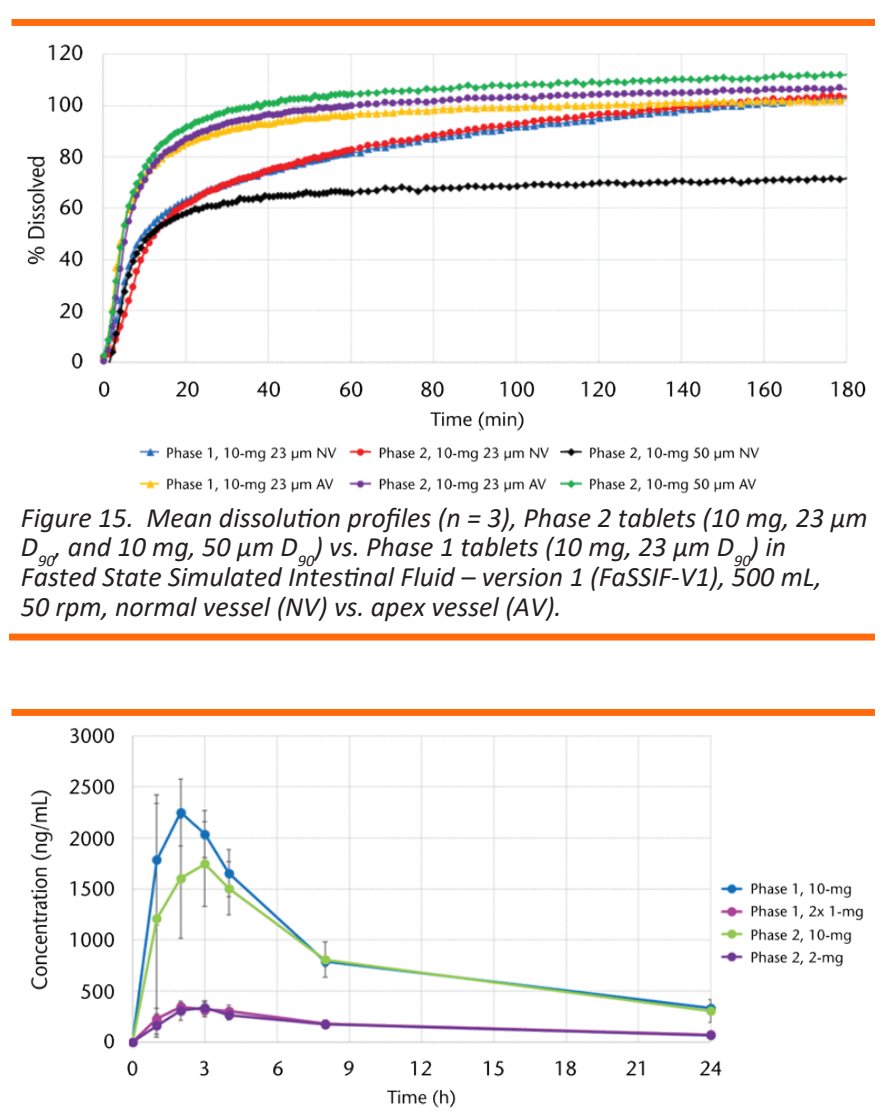

Figure 16. Dog PK $(n=4)$ exposure of Phase $1(2 \times 1$ - and 10-mg) and Phase 2 (2- and 10-mg) tablets. The API particle sizes for all tablets used for the dog study are $23 \mu \mathrm{m} D_{90^{*}}$.

\section{RESULTS AND DISCUSSION OF INTERLABORATORY STUDY}

The information recorded for all participants as part of the protocol is summarized in Table 4. Most partners used cell-based UV detectors either with manual or semi-automated sampling; three partners used in situ fiber optic detection, which resulted in not requiring any filtration. The majority of partners used a $0.45-\mu \mathrm{m}$ PVDF 
filter but some partners used alternate membranes; all but one partner who utilized filters used a cannula prefilter alongside a syringe filter, whereas only partner 8 utilized only a cannula filter. The dimensions reported for inner diameter ranged from 100-104 mm, which is within the USP range of 98-106 mm. It was obvious that the vessels for the Sotax ATXtend and the Erweka systems were produced at a higher target inner diameter than both the Sotax AT7 system and the other manufacturers. Deviation of the apex from the center of the vessel was on average between 0 and $1 \mathrm{~mm}$ with a maximum deviation of $2 \mathrm{~mm}$ observed across all vessels measured. The height between the apex and paddle for most systems could be set to $10 \mathrm{~mm}$, one system the Sotax AT7 was not adjusted and gave a range of between 7 and $12 \mathrm{~mm}$.

Data from all partners are plotted in Figure 17 by bath manufacturer. This figure shows an overall low degree of variability in the dissolution across all 11 partners, with a range of $82-100 \%$ dissolved across 132 measurements at the 30-min time point, and an overall mean and \% coefficient of variation (CV) of $92 \%$ and $3.3 \%$, respectively. The means at the 30-min time point range from $88 \%-$ $94 \%$ across the 11 partners $(n=12)$ with a range of $\% \mathrm{CV}$ from $1.0-4.0 \%$ (Table 4).

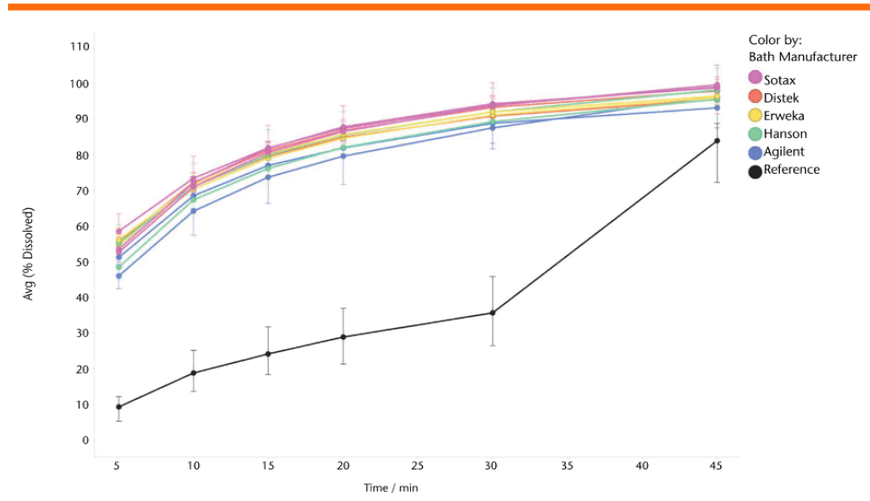

Figure 17. All dissolution data from interlaboratory study plotted by bath manufacturer (error bars represent maximum and minimum values). Avg, average.

\begin{tabular}{|c|c|c|c|c|c|c|c|c|c|}
\hline Partner & $\begin{array}{l}\text { Bath } \\
\text { Model }\end{array}$ & $\begin{array}{c}\text { Vessel } \\
\text { Manufacturer }\end{array}$ & UV Type & $\begin{array}{l}\text { Cannula } \\
\text { Filter }\end{array}$ & $\begin{array}{l}\text { Syringe } \\
\text { Filter }\end{array}$ & $\begin{array}{c}\text { Inner } \\
\text { Diameter } \\
\text { (mm) Mean } \\
\text { (Minimum- } \\
\text { Maximum) }\end{array}$ & $\begin{array}{c}\text { Apex } \\
\text { Deviation } \\
\text { (mm) Mean } \\
\text { (Minimum- } \\
\text { Maximum) }\end{array}$ & $\begin{array}{c}\text { Height } \\
\text { (mm) Mean } \\
\text { (Minimum- } \\
\text { Maximum) }\end{array}$ & $\begin{array}{c}\text { Mean \% } \\
\text { Dissolved at } 30 \\
\text { min (\%Relative } \\
\text { Standard } \\
\text { Deviation) }\end{array}$ \\
\hline 1 & $\begin{array}{l}\text { Agilent } \\
\text { 708-DS }\end{array}$ & Agilent & Cell & Yes & $\begin{array}{l}0.45 \mu \mathrm{m} \\
\text { PVDF }\end{array}$ & $101(101-101)$ & $0(0-0)$ & $10(10-10)$ & $89(2.6)$ \\
\hline 2 & $\begin{array}{c}\text { Distek } \\
2500 \\
\text { Select }\end{array}$ & Distek & Fiber & - & - & $101(101-101)$ & $0(0-1)$ & $10(10-10)$ & $93(1.9)$ \\
\hline 3 & $\begin{array}{c}\text { Sotax } \\
\text { ATXtend }\end{array}$ & Sotax & Cell & Yes & $\begin{array}{l}0.45 \mu \mathrm{m} \\
\text { PVDF }\end{array}$ & $103(103-104)$ & $0(0-1)$ & $10(9-11)$ & $94(1.0)$ \\
\hline 4 & $\begin{array}{l}\text { Agilent } \\
\text { 708-DS }\end{array}$ & Agilent & Cell & Yes & $\begin{array}{l}0.45 \mu \mathrm{m} \\
\text { PVDF }\end{array}$ & $101(100-101)$ & $1(0-1)$ & $10(10-10)$ & $88(4.0)$ \\
\hline 5 & $\begin{array}{l}\text { Hanson } \\
\text { Vision G2 } \\
\text { Elite } 8\end{array}$ & Hanson & Cell & Yes & $\begin{array}{l}0.45 \mu \mathrm{m} \\
\text { Nylon }\end{array}$ & $101(101-101)$ & $0(0-0)$ & $10(10-10)$ & $89(1.4)$ \\
\hline 6 & $\begin{array}{l}\text { Distek } \\
7100\end{array}$ & Distek & Fiber & - & - & $101(101-101)$ & $0(0-0)$ & $10(10-10)$ & $91(2.1)$ \\
\hline 7 & $\begin{array}{c}\text { Hanson } \\
\text { Vision G2 } \\
\text { Elite } 8 \\
\end{array}$ & QLA & Fiber & - & - & $101(101-101)$ & $1(0-1)$ & $10(10-10)$ & $92(3.0)$ \\
\hline 8 & $\begin{array}{l}\text { Erweka } \\
\text { DT820 }\end{array}$ & Erweka & Cell & Yes & - & $104(103-104)$ & $1(1-2)$ & $10(10-10)$ & $92(1.1)$ \\
\hline 9 & $\begin{array}{l}\text { Sotax AT7 } \\
\text { Smart }\end{array}$ & Sotax & Cell & No & $\begin{array}{l}0.45 \mu \mathrm{m} \\
\text { PVDF }\end{array}$ & $100(100-101)$ & $0(0-0)$ & $10(7-12)$ & $94(3.4)$ \\
\hline 10 & $\begin{array}{l}\text { Sotax } \\
\text { ATXtend }\end{array}$ & Sotax & Cell & Yes & $\begin{array}{l}0.45 \mu \mathrm{m} \\
\text { PTFE }\end{array}$ & $104(104-104)$ & $1(0-1)$ & NM & $94(1.6)$ \\
\hline 11 & $\begin{array}{l}\text { Erweka } \\
\text { DT720 }\end{array}$ & Erweka & Cell & Yes & $\begin{array}{c}0.45 \mu \mathrm{m} \\
\text { PVDF }\end{array}$ & $103(101-104)$ & $1(0-1)$ & $10(10-10)$ & $91(1.5)$ \\
\hline Reference & $\begin{array}{l}\text { Agilent } \\
\text { 708-DS }\end{array}$ & Agilent & Cell & Yes & $\begin{array}{l}0.45 \mu \mathrm{m} \\
\text { PVDF }\end{array}$ & $101(100-101)$ & - & - & $36(22.5)$ \\
\hline
\end{tabular}

PVDF = polyvinylidene difluoride; $P T F E=$ polytetrafluoroethylene; $N M=$ not measured. 
The reference profile using the standard vessel in Figure 17 ( $n=6$ vessels) shows a mean of $36 \%$ and a $\%$ CV of $22.5 \%$ at the 30- min time point. This cannot be compared with the acceptance criterion for a traditional PVT test, as PVT is done only at a single time point, whereas to compare with the apex vessel data, a profile was collected. The vessels that showed a high percentage dissolved were the tablets observed to land off center and dissolve more prior to the cone forming. This indicates how sensitive the dissolution of prednisone is to the position of the tablets in the conventional vessel and how the apex vessel helps normalize that variability by removing the propensity for coning.

By using an $f_{2}$ calculation between the fastest and slowest apex vessel profile to give the highest degree of difference observed gives a calculated $f_{2}$ of 50.8 , which indicates profile similarity using all standard $f_{2}$ rules around variability and time points to include once $85 \%$ is reached (5-20-min time points used), if all time points are used, then $f_{2}$ increases to 53.9. In addition, a statistical equivalency comparison was carried out between these profiles, a $90 \%$ confidence interval for the difference in means at a 30-min span from 3.9-8.6\%. Furthermore, the difference between the mean apex vessel profile and the conventional vessel reference at all time points far exceeds $\pm 10 \%$, indicating that the profiles between conventional and apex vessels are dissimilar.

The principal component analysis carried out on the mean dissolution profiles led to two key principal components, the first of which relates to the vertical shift of the curve (explaining $86 \%$ of the variability) and the second describing the shape of the curve (explaining $12 \%$ of the variability). The scores plot in Figure 18 plots principal component 2 (t[2]) versus principal component 1 (t[1]). Partners that fall close together in the plot will have dissolution profiles matching most closely on both these aspects (Figure 17). None of the points fall outside of the $95 \%$ confidence ellipse, indicating there is no evidence of any outlying partners.

Each of the vessel-specific factors were explored in turn by analysis of covariance, with 30-min dissolution data as the response and partner as categorical factor. None of the vessel-specific factors were found to be statistically significant ( $p=0.73$ for internal diameter, $p=0.88$ for apex deviation, $p=0.51$ for height).

The mean dissolution data are plotted in Figure 19 distinguishing the bath manufacturer and vessel manufacturer by color and shape, respectively. A one-way analysis of variance was fitted to these data adjusting for multiple comparisons [Tukey-Kramer Honest Significant Difference (HSD) test]. This analysis reveals a statistically significant difference between Sotax and Agilent with respect to bath manufacturer $(p<0.05)$. However, it should be noted that the magnitude of difference in mean profile is small and unimportant scientifically.

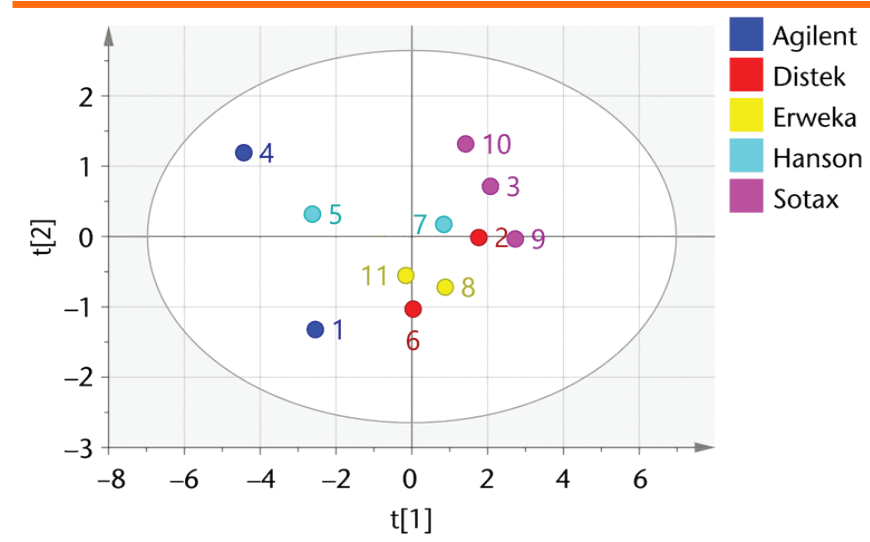

Figure 18. Principal component analysis on mean dissolution profilesscores plot.

The differences do not appear to be related to vessel dimensions, as the Sotax AT7 Smart had a mean diameter of $100 \mathrm{~mm}$, which was the lowest of the study, and the Sotax ATXtend, which had a higher mean diameter of 103-104 mm. Additionally, the Erweka system also had a slightly larger inner diameter of $103 \mathrm{~mm}$ but did not show any difference from other partners. Across the three partners who used Sotax, one used the hollow shaft sampling and the other two used cannula for sampling so sampling method did not explain the difference. It is worth noting that the fastest profiles were produced in the Sotax AT7 Smart bath where the partners did not adjust the paddle to an apex to paddle height of 10 $\mathrm{mm}$, although the analysis of the data did not indicate any correlation between the set height and the percent dissolved. This suggests that a $10-\mathrm{mm}$ setting with a tolerance of $2 \mathrm{~mm}$ would be acceptable as a specification. This data set was also produced across two completely different baths, which was a deviation from the agreed protocol, albeit with the same autosampler serving both baths. In the authors' opinion, the most likely differences are related to subtle differences in autosampler designs and settings between manufacturers. This is supported by one partner who used a flow-through UV system that the various prime and purge settings on the autosamplers were not set up correctly and so the sample analyzed in the cell did not correspond to the sample at the time of pull. This data was recollected with adjusted settings and was found to be within trend. Overall, the practical impact of using different manufacturer systems and corresponding apex vessels was low, and therefore, any 


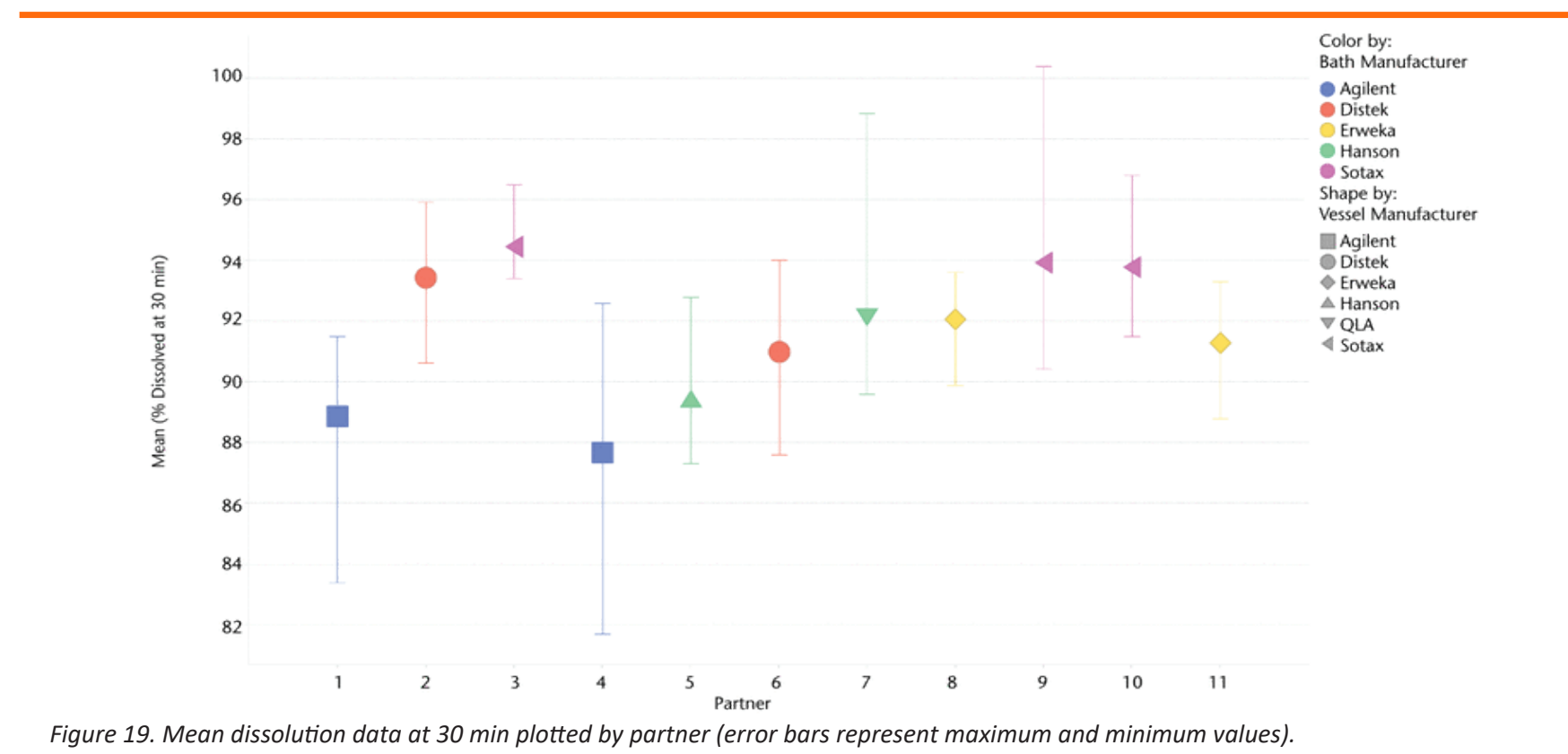

Figure 19. Mean dissolution data at 30 min plotted by partner (error bars represent maximum and minimum values).

proposed specification is suitable to encompass the ranges observed across all five major manufacturers.

\section{RESULTS AND DISCUSSION OF CFD STUDY}

Figure 20 illustrates the simulated hydrodynamics in an apex vessel, specifically based on the dimensions of the Agilent apex vessel. Colors indicate the velocity magnitude, ranging from 0 at the wall to the paddle tip speed of $0.2 \mathrm{~m} / \mathrm{s}$, and vectors indicate the direction of fluid motion. There is a strong tangential flow throughout the vessel due to the motion of the paddle. A small low velocity stagnant region is observed between the top of the apex and the paddle, but good fluid flow is generated at the lowest positions in the vessel due to the central apex. This is consistent with experimental observations where a small amount of material can sit at the top of the apex, but the base of the vessel is clear of deposition.

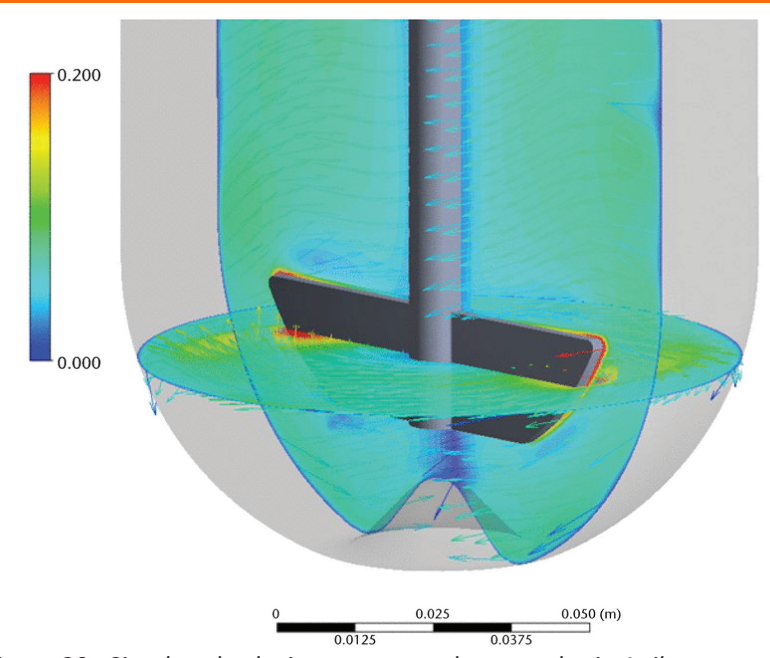

Figure 20. Simulated velocity contour and vector plot in Agilent apex vessel. The color scale indicates velocity magnitude in $\mathrm{m} / \mathrm{s}$.
The shape of the apex will direct the tablet to sink into the off-center region between the apex and side wall, which exhibits a consistent fluid velocity between approximately 7 and $8 \mathrm{~cm} / \mathrm{s}$ (at a paddle speed of $50 \mathrm{rpm}$ ). In contrast, a standard vessel exhibits a heterogeneous velocity distribution at the base, with a stagnant region below the impeller and increasing fluid velocity moving outward. The fluid velocity at the base of a standard vessel corresponding to the radial position where the tablet would land in an apex vessel is calculated to be very similar. Therefore, a tablet that lands in this region would experience similar hydrodynamics and be expected to give similar dissolution profiles between both vessels.

One method of quantifying the hydrodynamics and mixing potential within the vessel is to calculate the shear rate distribution. Figure 21 shows a comparison of the simulated shear rate distributions across the different vessel geometries for the region around the paddle and below. This shows the majority of the volume in this region experiencing low to moderate shear rates of approximately $10 \mathrm{~s}^{-1}$ and below. A smaller volume experiences much higher shear rates between 10 and 100 $\mathrm{s}^{-1}$, primarily near the paddle and the walls of the vessel, including the apex. All manufacturers' vessels exhibit very similar shear rate distributions with minor differences observed at low shear rates. To further support this, the volume mean shear rates are shown in Figure 22. All vessels show very little difference in mean shear rate, indicating that the small geometric differences have very little influence on the overall hydrodynamic behavior of the vessel. 


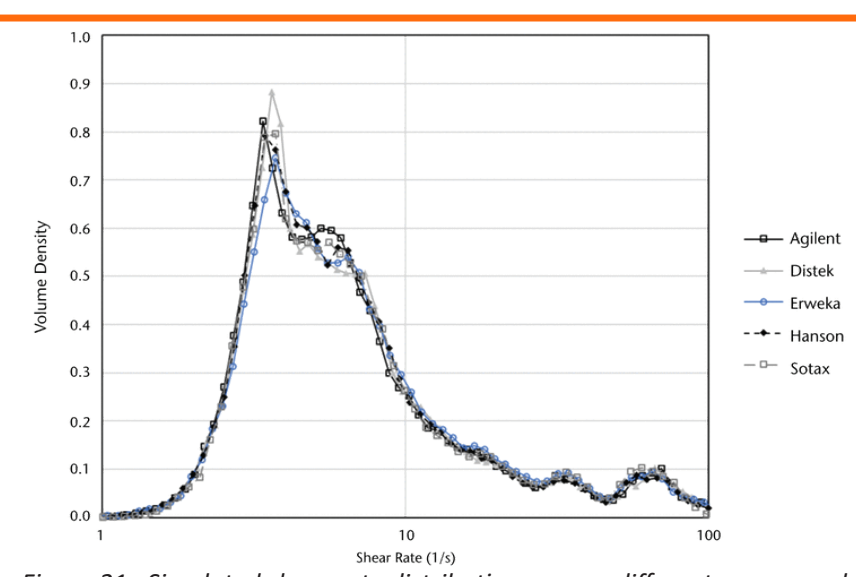

Figure 21. Simulated shear rate distributions across different apex vessel geometries for the region around and below the paddle.

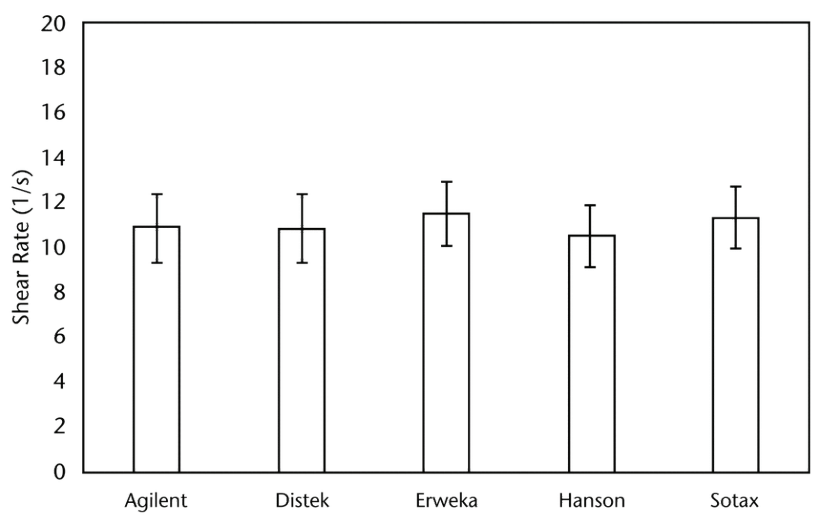

Figure 22. Simulated volume mean shear rates in the region around and below the paddle for each vessel geometry. Error bars indicate the variance of the distributions.

\section{PROPOSED SPECIFICATION OF APEX VESSEL}

As indicated in the Introduction, two major barriers to more prevalent utilization of apex vessels are the lack of specifications for these vessels to ensure equivalence among different manufacturers of apex vessels and agreed procedures for qualification and utilization of a dissolution system incorporating these vessels. In addressing this concern, the AAPS IVRDT Community Instrumentation Sub-Group collaborated with our colleagues from the IQ to help convert the results from this interlaboratory study into a set of recommended specifications and qualification and implementation procedures for apex vessels.

As with any specified compendial component, the idea was to generate a set of specifications that will guarantee functionality of the component for the intended purpose, allow component manufacturers to measure, and certify compliance with said specification.

Similarly, there is a guidance for the implementation of these components as part of a validated dissolution system.
At the core of specifying what constitutes an appropriate apex vessel is the fact that these are still dissolution vessels, only with the addition of the apex at their base. That means that there is no compelling reason (nor was any exhibited in the study) to alter the existing standard 1 - $L$ vessel height and inner diameter specifications.

- Apex Dimensions: An apex vessel will have a true cone protruding into the volume of the vessel featuring a conical top with no flat area. Although there are multiple ways to specify the apex at the bottom of an apex vessel, the most reproducible, measurable, and relevant as demonstrated in this study seem to be the apex height, the apex cone angle, and the deviation from the center of the apex, shown in Figure 23.

- Apex Height: This proposal is for an apex that is $15 \pm 2.5 \mathrm{~mm}$ in height. This is measured from the top of the apex on the interior of the vessel to the point where the vessel meets a flat surface on the exterior of the vessel. The reason for selecting the external base of the apex versus the interior base is that the latter depends on the method used to generate the apex and can result in vague values. The total allowed variation of $\pm 2.5 \mathrm{~mm}$ is comparable to the range within the vessels from various manufacturers used in the study, which showed no correlation with the height over the range spanned.

- Apex Cone Angle: An apex cone angle specification of $87^{\circ} \leq \theta \leq 93^{\circ}$ is recommended. The angle $\theta$ is measured on the external side

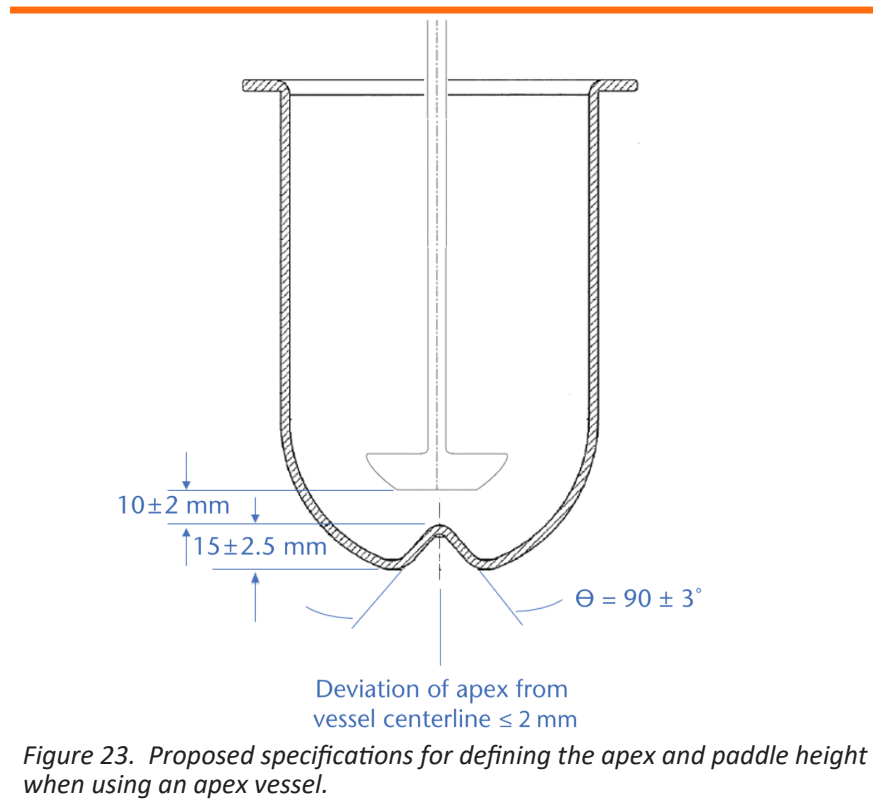

Dissolution

NOVEMBER 2021 Technologies www.dissolutiontech.com 
of the apex. The choice of the external side is predicated by the ease and accuracy of the measurement possible. The proposed range is supported by the existing range of vessels used in the study as well as the CFD modeling, which shows a uniform flow field of a greater extent near the modeled apex.

- Apex Centering: A tolerance of $\pm 2 \mathrm{~mm}$ of the apex centering relative to the vessel center is suggested. The choice is again supported by both a lack of correlation with the deviation of the vessels used in the study and the CFD results that show a significantly larger low velocity flow area directly under the approximately $10-\mathrm{mm}$ paddle center shaft than the deviation proposed.

\section{QUALIFICATION OF APEX VESSEL}

The proposed recommendation is that any dissolution apparatus that will be used with apex vessels will first be qualified according to the company protocols regarding enhanced mechanical and/or PVT qualification. Subsequent to the completion of the qualification with standard vessels, these will be replaced with validated apex vessels. The paddle height will then be readjusted (and be set for all subsequent measurements) to $10 \pm 2$ $\mathrm{mm}$ above the apex. The \pm 2 -mm tolerance is borne out by both the CFD modeling and the lack of correlation with the AT7 Smart data that utilized heights wider than the proposed range. For Sotax systems where the paddle height is not normally adjustable, a set of adjustable paddles are available, which were developed for paddle over disk systems. A check device is available for all high-head bath systems, but a new check device will be required for low-head bath systems.

\section{CONCLUSION}

The purpose of the dissolution method is to measure rate and extent of release and should be sensitive to factors that matter and insensitive to factors that do not. No method should measure artifacts and because the gastro-intestinal (GI) tract is not a hemispherical dissolution vessel with a paddle and the dosage form does not reside in one position during gastric transit, then coning is not expected in vivo. For this reason, efforts should be made to eliminate the artifact of coning during dissolution experiments. Using real industrial examples, the benefits of utilizing the apex vessel to overcome coning have been demonstrated. Case studies have demonstrated that by removing coning improved links to in vivo data are possible, as are more robust and meaningful discriminatory methods. Finally, formulation bridging using in vitro tools can be streamlined when in vitro artifacts are removed.

By conducting an interlaboratory study across all major dissolution bath manufacturers and industrial partners alongside a CFD modeling approach, an assessment of the performance difference across the participating baths was undertaken and showed that small differences in vessels made little significant impact on the hydrodynamics or dissolution performance of the prednisone tablet dissolution across partners. Finally, the dissolution manufacturers agreed on a specification and qualification procedure for the manufacture and use of apex vessels.

It is the authors' intent by publishing the case studies, interlaboratory study, and CFD modeling of the apex vessel that this will lead to greater acceptance of the apex vessel and will be considered for a more prominent inclusion in future pharmacopoeial chapters such as USP chapter 〈1092), and ultimately inclusion into (711) in the USP and other harmonized pharmacopoeia as an alternative vessel to the standard 1-L vessel.

\section{ACKNOWEDGEMENTS}

This Stimuli article was developed with the support of the International Consortium for Innovation and Quality in Pharmaceutical Development (IQ, www.iqconsortium. org) and the American Association of Pharmaceutical Scientists (AAPS).

\section{REFERENCES}

1. Pepin XJH, Flanagan TR, Holt DJ, Eidelman A, Treacy D, Rowlings CE. Justification of drug product dissolution rate and drug substance particle size specifications based on absorption PBPK modeling for lesinurad immediate release tablets. Mol Pharm. 2016;13(9):3256-3569.

2. Hermans A, Abend AM, Kesisoglou F, Flanagan T, Cohen MJ, Diaz DA, et al. Approaches for establishing clinically relevant dissolution specifications for immediate release solid oral dosage forms. AAPS J. 2017;19(6):1537-1549.

3. Baxter JL, Kukura J, Muzzio FJ. Hydrodynamics-induced variability in the USP apparatus II dissolution test. Int J Pharm. 2005;292(12):17-28.

4. Bai G, Armenante PM, Plank RV, Gentzler M, Ford K, Harmon P. Hydrodynamic investigation of USP dissolution test apparatus II. J Pharm Sci. 2007;96(9):2327-2349.

5. Higuchi M, Yoshihashi Y, Tarada K, Sugano K. Minimum rotation speed to prevent coning phenomena in compendium paddle dissolution apparatus. Eur J Pharm Sci. 2014;65:74-78.

6. Beckett AH, Quach TT, Kurs GS. Improved hydrodynamics for USP apparatus 2. Dissolution Technol. 1996;3(2):7-18.

7. Collins CC, Nair RR. Comparative evaluation of mixing dynamics 
in USP apparatus 2 using standard USP vessels and PEAK vessels. Dissolution Technol. 1998;5(2):17-20.

8. Baxter JL, Kukura J, Muzzio FJ. Shear-induced variability in the United States Pharmacopeia apparatus 2: modifications to the existing system. AAPS J. 2006;7(4):857-864.

9. Beckett $\mathrm{AH}$, Swon JE, Hofer $\mathrm{HZ}$, inventors; VanKel, assignee. Device maximising dispersion of aggregate in liquid diluent. U.S. Patent No. 5,700,087A. 1997.

10. Mirza T, Joshi Y, Liu Q, Vivilecchia R. Evaluation of Dissolution Hydrodynamics in the USP, Peak and flat-bottom vessels using different solubility drugs. Dissolution Technol. 2005;1(1):11-16.

11. Nithyanandan P, Deng G, Brown W, Manning R, Wahab S.
Evaluation of the sensitivity of USP prednisone tablets to dissolved gas in the dissolution medium using USP Apparatus 2. Dissolution Technol. 2006;13(3):15-18.

12. Shah VP, Tsong Y, Sathe P, Liu J-P. In vitro dissolution profile comparison-statistics and analysis of the similarity factor, $f_{2}$. Pharm Res. 1998;15(6):889-896.

13. Hopgood M, Reynolds G, Barker R. Using computational fluid dynamics to compare shear rate and turbulence in the TIMautomated gastric compartment with USP apparatus II. J Pharm Sci. 2018;107(7):1911-1919.

14. ICH Harmonized Guideline: Biopharmaceutics Classification System-Based Biowaivers M9. 2019. 